\title{
The Hamburg/ESO R-process Enhanced Star survey (HERES) ${ }^{\star}$
}

\section{The $r+s$ star HE 1405-0822 ${ }^{\star \star}$}

\author{
W. Y. Cui ${ }^{1,2}$, T. Sivarani ${ }^{3}$, and N. Christlieb ${ }^{1}$ \\ 1 Zentrum für Astronomie der Universität Heidelberg, Landessternwarte, Königstuhl 12, 69117 Heidelberg, Germany \\ e-mail: cui@lsw.uni-heidelberg.de; N.Christlieb@lsw.uni-heidelberg.de \\ 2 Department of Physics, Hebei Normal University, Nanerhuan dong Road 20, 050024 Shijiazhuang, PR China \\ e-mail: cuiwenyuan@hebtu.edu.cn \\ 3 Indian Institute of Astrophysics, 560034 Bangalore, India \\ e-mail: sivarani@gmail.com
}

Received 29 March 2013 / Accepted 13 August 2013

\section{ABSTRACT}

\begin{abstract}
Aims. The aim of this study is a detailed abundance analysis of the newly discovered r-rich star HE 1405-0822, which has $[\mathrm{Fe} / \mathrm{H}]=-2.40$. This star shows enhancements of both $\mathrm{r}$ - and s-elements, $[\mathrm{Ba} / \mathrm{Fe}]=+1.95$ and $[\mathrm{Eu} / \mathrm{Fe}]=1.54$, for which reason it is called $\mathrm{r}+\mathrm{s}$ star.

Methods. Stellar parameters and element abundances were determined by analyzing high-quality VLT/UVES spectra. We used Fe I line excitation equilibria to derive the effective temperature. The surface gravity was calculated from the $\mathrm{Fe} / \mathrm{I} / \mathrm{Fe}$ II and Ti I/Ti II equilibria.

Results. We determined accurate abundances for 39 elements, including 19 neutron-capture elements. HE 1405-0822 is a red giant. Its strong enhancements of $\mathrm{C}, \mathrm{N}$, and s-elements are the consequence of enrichment by a former AGB companion with an initial mass of less than $3 M_{\odot}$. The heavy n-capture element abundances (including Eu, Yb, and Hf) seen in HE 1405-0822 do not agree with the r-process pattern seen in strongly r-process-enhanced stars. We discuss possible enrichment scenarios for this star. The enhanced $\alpha$ elements can be explained as the result of enrichment by supernovae of type II. Na and $\mathrm{Mg}$ may have partly been synthesized in a former AGB companion, when the primary ${ }^{22} \mathrm{Ne}$ acted as a neutron poison in the ${ }^{13} \mathrm{C}$-pocket.
\end{abstract}

Key words. stars: abundances - stars: AGB and post-AGB - stars: atmospheres - stars: chemically peculiar

\section{Introduction}

Elements beyond the iron group are believed to be mostly synthesized through neutron-capture (hereafter n-capture) processes, which consist of the rapid (r-) and the slow (s-) process. These are distinguished by the timescales for neutron captures relative to the $\beta$-decay timescales of the resulting nuclei (Burbidge et al. 1957). The s-process is generally assumed to take place in the asymptotic giant branch (AGB) phase of stars of low or intermediate mass. However, Pignatari et al. (2008) suggested that in metal-poor, fast-rotating stars (hereafter spin stars), the efficiency of the s-process is high enough to produce the strong overabundances of $\mathrm{Sr}, \mathrm{Y}$, and $\mathrm{Zr}$ observed in extremely metal-poor halo stars $([\mathrm{Fe} / \mathrm{H}]<-3.0)^{1}$, where the AGB stars do not have time to contribute. Using models of extremely metalpoor spin stars, Chiappini et al. (2011) successfully explained the large scatter in [Y/Ba] ratios in NGC 6522, the oldest globular cluster of the Milky Way. This large scatter is also seen in the most metal-poor halo stars.

Some explosive astrophysical events are usually accompanied by synthesis of the r-process elements, but the exact site(s)

* Based on observations collected at the European Southern Observatory, Paranal, Chile (Proposal numbers 170.D-0010G, and 170.D-0010J).

$\star \star$ Tables 5, 6 are available in electronic form at http://www . aanda.org

1 The standard spectroscopic notation is used, i.e., $[\mathrm{X} / \mathrm{H}]=$ $\log _{10}\left(N_{\mathrm{X}} / N_{\mathrm{H}}\right)_{\star}-\log _{10}\left(N_{\mathrm{X}} / N_{\mathrm{H}}\right)_{\odot}$, where $N_{\mathrm{X}}$ is the number density of atoms of the element $X$. of this process is still unclear (Sneden et al. 2008). Several possibilities have been suggested, including prompt explosions of core-collapse (Type II/Ibc) supernovae (Wanajo et al. 2003), neutron star mergers (Lattimer et al. 1977; Rosswog et al. 1999; Freiburghaus et al. 1999), neutrino-driven winds (Woosley et al. 1994; Wanajo et al. 2001), the accretion-induced collapse mechanism (AIC, Qian \& Wasserburg 2003; Cohen et al. 2003), and Type 1.5 supernovae (Iben \& Renzini 1983; Zijlstra 2004). More observational studies of r-process-enriched stars may shed light on this research field.

Recent studies indicate that there may be two separate r-processes, which are referred to as the main r-process, which is responsible for the creation of heavy n-capture elements with $Z \geq 56$ (Truran et al. 2002; Sneden et al. 2003), and a weak r-process for the light n-capture elements with $Z<56$ (Kratz et al. 2007; Wanajo \& Ishimaru 2006). In the strongly r-process enhanced stars, i.e. stars with $[\mathrm{Eu} / \mathrm{Fe}]>+1.0$ and $[\mathrm{Ba} / \mathrm{Eu}]<0$, (hereafter r-II stars, Beers \& Christlieb 2005), the abundance distribution of heavy n-capture elements does not vary significantly from star to star, and it agrees very well with the scaled solar system r-process distribution. Up to now, about ten r-II stars have been discovered, including CS 22892-052 (Sneden et al. 2003, 2009), CS 31082-001 (Hill et al. 2002), CS 31078-018 (Lai et al. 2008), HD 221170 (Ivans et al. 2006; Sneden et al. 2009), $\mathrm{BD}+17^{\circ} 3248$ (Cowan et al. 2002, 2011; Roederer et al. 2010b), CS 22953-003 (François et al. 2007), HE 1523-0901 (Frebel et al. 2007), HD 115444 (Westin et al. 2000; Sneden et al. 2009; Hansen \& Primas 2011), CS 29491-069 (Hayek et al. 2009), HE 1219-0312 (Hayek et al. 2009), and HE 2327-5642 
(Mashonkina et al. 2010). Studies on these r-II stars confirmed the universal pattern of the main r-process (Cowan et al. 2011).

Unlike the main r-process pattern seen in r-II stars, there are significant deviations between the light n-capture elements ( $37 \leq Z \leq 47$, i.e., from $\mathrm{Rb}$ to $\mathrm{Ag}$ ) in r-II stars and the scaled solar system r-process pattern. This implies that multiple r-process sites (Wasserburg et al. 1996; Wasserburg \& Qian 2000; Qian \& Wasserburg 2000, 2001, 2002) or the same core-collapse supernovae but different epochs or regions (Cameron 2001, 2003) are probably responsible for the solar r-process distribution. Some r-process-poor stars, such as HD 122563 and HD 88609, show a high excess of light n-capture elements (e.g., Sr, Y and $\mathrm{Zr}$ ), but no enrichment of the heavy ones (e.g., Ba, Eu), which indicates that the weak r-process plays a dominant role in producing their abundance patterns (Honda et al. 2006, 2007; Izutani et al. 2009). In fact, a combination of processes, such as the weak r-process and the main r-process, is more efficient in reproducing the observed abundances of light $n$-capture elements for many such types of stars (Zhang et al. 2010; Roederer et al. 2010b,a; Arcones \& Montes 2011; Cowan et al. 2011). Though the n-capture element distribution in CS 22892-052 is not similar to that of most metal-poor stars, Cowan et al. (2011) points out that the r-process enrichment in the early Galaxy is common because of the presence of $\mathrm{Sr}, \mathrm{Ba}$, etc. in nearly all metal-poor stars that do not show s-process enhancements.

Some metal-poor s-rich stars at the same time show a strong enrichment of Eu and other heavy neutron-capture elements, which in the solar system are predominantly produced by the r-process. These stars are commonly referred to as $\mathrm{r}+\mathrm{s}$ stars (Beers \& Christlieb 2005). Their s-process enrichment is usually attributed to mass transfer (by wind accretion or Roche-lobe overflow) from a former AGB companion, which now most likely is a white dwarf. In fact, many s-rich stars have been found to be binaries (McClure et al. 1980; McClure \& Woodsworth 1990; North et al. 2000; Barbuy et al. 2005; Lucatello et al. 2006, 2009). A variety of scenarios for explaining the abundance patterns of $r+s$ stars have been suggested (see, e.g., Jonsell et al. 2006, and references therein), but so far, none of them can coherently explain all observational phenomena. Studies of additional $\mathrm{r}+\mathrm{s}$ stars may shed some lights on the general questions about the $r$ - and s-processes, such as the site or sites of the r-process and the relative contribution of these two processes under metalpoor conditions.

To identify and study strongly r-process enhanced metalpoor stars, i.e., r-II stars, the Hamburg/ESO R-process Enhanced Star survey (HERES) has been carried out (Christlieb et al. 2004). First, the metal-poor candidates were selected in the digital spectra database of the Hamburg/ESO objective-prism survey (HES; Wisotzki et al. 2000). A detailed description of the selection method and the method with which the metal-poor nature of these candidates was confirmed based on their moderateresolution $(\Delta \lambda \sim 2 \AA)$ follow-up spectroscopy can be found in Christlieb et al. (2008). During the course of HERES, "snapshot" spectra (i.e., spectra with $R=\lambda / \Delta \lambda=20000$ and a typical signal-to-noise ratio of $S / N=50$ ) were obtained with the Very Large Telescope (VLT) Unit Telescope 2 (UT2) and the Ultraviolet-Visual Echelle Spectrograph (UVES) for several hundred confirmed metal-poor stars. HE 1405-0822, the star studied here, is one of them. It is a red giant star with a metallicity of $[\mathrm{Fe} / \mathrm{H}] \sim-2.4$, in which $\mathrm{Eu}$ and $\mathrm{Ba}$ are both enhanced. Therefore, higher quality spectra of this star were obtained with VLT/UVES (for details, see Sect. 2). Our detailed abundance analysis is based on these spectra.
Table 1. Photometry and astrometry of HE 1405-0822.

\begin{tabular}{lc}
\hline \hline $\mathrm{RA}(\mathrm{J} 2000.0)$ & $14 \mathrm{~h} 07 \mathrm{~m} 42.9 \mathrm{~s}$ \\
$\operatorname{Dec}(\mathrm{J} 2000.0)$ & $-08^{\circ} 36^{\prime} 14.3^{\prime \prime}$ \\
$V$ & $13.998 \pm 0.003$ \\
$B-V$ & $0.772 \pm 0.008$ \\
$V-R$ & $0.407 \pm 0.005$ \\
$V-I$ & $0.827 \pm 0.005$ \\
\hline
\end{tabular}

Notes. The photometry was taken from Beers et al. (2007).

Table 2. Barycentric radial velocities of HE 1405-0822.

\begin{tabular}{lcc}
\hline \hline $\begin{array}{l}\text { MJD } \\
\text { [days }]\end{array}$ & $\begin{array}{c}R V \\
{\left[\mathrm{~km} \mathrm{~s}^{-1}\right]}\end{array}$ & $\begin{array}{c}\sigma \\
{\left[\mathrm{km} \mathrm{s}^{-1}\right]}\end{array}$ \\
\hline 52762.213 & 124.01 & 0.55 \\
53451.178 & 138.13 & 0.17 \\
53451.214 & 138.43 & 0.53 \\
53451.251 & 137.96 & 0.35 \\
53451.288 & 138.97 & 0.71 \\
53451.325 & 138.15 & 0.45 \\
\hline
\end{tabular}

\section{Observations and data reduction}

Astrometry and photometry of HE 1405-0822 are listed in Table 1; the photometry was taken from Beers et al. (2007). High-quality spectra of this object were obtained during the night of 22 March 2005 with VLT-UT2 and UVES in dichroic mode. The standard setting BLUE346+RED580 was used, resulting in a spectral coverage of $3046-3863 \AA$ in the blue arm, and $4781-6809 \AA$ in the red arm, with a gap between the two CCD detectors causing a gap in wavelength coverage of 5757-5833 $\AA$. Each spectrum has an exposure time of about $1 \mathrm{hr}$, and the total is $5 \mathrm{hr}$. The slit width in both arms was set to $0.8^{\prime \prime}$, so that a resolving power of $R=40000$ was achieved. In the wavelength gap from $3850 \AA$ to $4795 \AA$ we used the snapshot spectrum, which was obtained with VLT/UVES on 3 May 2003.

The geocentric radial velocities of the individual spectra were determined by fitting Gaussian profiles to $\sim 10$ moderately strong, clean lines. Then the spectra were shifted to the rest frame. The individual higher-resolution spectra were coadded in an iterative procedure, in which pixels affected by cosmicray hits or CCD defects were rejected by $\kappa \sigma$-clipping. The final coadded spectrum was obtained by computing the weighted mean of the individual spectra. In this coadded spectrum, the average $\mathrm{S} / \mathrm{N}$ per pixel is $S / N \sim 34$ from 3200 to $3800 \AA$ at Blue arm. The red-arm spectrum has $S / N>100$ per pixel throughout the covered wavelength range from 4800 to $6700 \AA$. The snapshot spectrum has $S / N \sim 50$ per pixel at $4100 \AA$.

The barycentric radial velocities of HE 1405-0822 and the observation epochs are listed in Table 2. The difference of the radial velocity between the snapshot spectrum, acquired at MJD = 52762.213 , and the higher-resolution spectra obtained 659 days later, is about $19 \mathrm{~km} \mathrm{~s}^{-1}$. This highly significant radial velocity variation is a strong indication that HE 1405-0822 is a member of a binary system. Radial velocity monitoring over several years will be needed to determine the period and orbital parameters of the system.

\section{Abundance analysis}

Our abundance analysis was carried out in local thermodynamic equilibrium (LTE) conditions. Most of the Fe-peak and 
Table 3. Stellar parameters of HE 1405-0822.

\begin{tabular}{lccccc}
\hline \hline Color/source/method & $\begin{array}{c}\text { Value } \\
{[\mathrm{mag}]}\end{array}$ & $\begin{array}{c}T_{\text {eff }} \\
{[\mathrm{K}]}\end{array}$ & $\log g$ & {$[\mathrm{Fe} / \mathrm{H}]$} & $\begin{array}{c}v_{\text {micro }} \\
{\left[\mathrm{km} \mathrm{s}^{-1}\right]}\end{array}$ \\
\hline$B-V$ & 0.772 & 4264 & & & \\
$V-R$ & 0.407 & 5321 & & & \\
$V-I$ & 0.827 & 5689 & & & \\
$R-I$ & 0.420 & 5305 & & & \\
Barklem et al. (2005) & & 5392 & 2.16 & -2.27 & 1.90 \\
Fe I \& Fe II lines & & 5220 & 1.70 & -2.40 & 1.88 \\
Adopted & & 5220 & 1.70 & -2.40 & 1.88 \\
\hline
\end{tabular}

$\alpha$ abundances were determined by means of equivalent width measurements. The analysis was restricted to lines with equivalent widths more narrow than $100 \mathrm{~m} \AA$ to avoid saturated lines and potential fitting errors of Gaussian line profiles due to damping wings that begin to appear at approximately this line strength. For the other elements, the spectrum synthesis method was used, employing the current version of the spectrum synthesis code (turbospectrum; Alvarez \& Plez 1998). The snapshot spectrum was used only for the abundances of crucial lines (e.g., $\mathrm{Sr}, \mathrm{CH}, \mathrm{CN}, \mathrm{Eu}$ ), which are not present in the high-resolution UVES spectrum. The abundance error is large for the snapshot spectrum for a given $\mathrm{S} / \mathrm{N}$ because of the lower resolution.

\subsection{Stellar parameters and model atmosphere}

An initial estimate of $T_{\text {eff }}$ was determined from broad-band optical and near-infrared colors, using the calibrations of Alonso et al. (1996) for $[\mathrm{Fe} / \mathrm{H}]=-2.0$. The transformation of 2MASS to TCS photometric system was the same as was used in Sivarani et al. (2004). We adopted a reddening of $E(B-V)=0.037$ (Schlegel et al. 1998). The resulting effective temperatures were used as an initial guess for the optimization routine, as in Barklem et al. (2005). We additionally refined the estimate using the line analysis procedure for the Fe I and Ti I lines.

The optimization routine by Barklem et al. (2005) determines the microturbulence spectroscopically. The method does not independently estimate $T_{\text {eff }}$ and $\log g$. It finds a minimum chi-square solution by fitting several weak metallic lines. However, the lines that are sensitive to $\log g$ are strong neutral and ionized lines. Hence there is a possibility of degeneracy between $T_{\text {eff }}$ and $\log g$. Therefore our additional refinement was to independently estimate $T_{\text {eff }}, \log g$, and the microturbulence velocity using the Fe I and Ti I lines. $T_{\text {eff }}$ was estimated from the Fe I and Ti I lines. $T_{\text {eff }}$ was determined from the Fe I lines by choosing a $T_{\text {eff }}$ that does gives no trend between the derived Fe abundance and its lower excitation potential of the Fe I line. There are very few Ti lines, however, therefore we used the Ti I lines only for a consistency check. The values obtained with the different methods are listed in Table 3. The surface gravity was derived from the $\mathrm{Fe} \mathrm{I} / \mathrm{Fe}$ II ionization equilibrium, and a consistency check was made using Ti I/Ti II ionization equilibrium. The microturbulence was determined by requiring that the abundances derived from the Fe I lines be independent of the measured equivalent widths.

We employed OSMARCS model atmospheres (see Gustafsson et al. 2003 and references therein). Because overabundances of $\mathrm{C}, \mathrm{N}$, and $\mathrm{O}$ may modify the temperature and density structure of the atmosphere, we used a model atmosphere tailored for HE 1405-0822, taking into account its enhancement in carbon, nitrogen, and oxygen.

\subsection{Line selection and atomic data}

We used the $\mathrm{CH}$ nd $\mathrm{CN}$ molecular line list compiled by Plez et al. (2005). The $\mathrm{NH}$ and $\mathrm{C}_{2}$ molecular line lists were taken from the Kurucz database ${ }^{2}$.

The line data for $\mathrm{Pb}$ were taken from Van Eck et al. (2003). McWilliam et al. (1995) pointed out that hyperfine splitting (HFS) has the effect of desaturating strong lines. Hence it is very important to perform HFS for strong lines. We included HFS and isotopic fractions as given in Van Eck et al. (2003). We detected seven Ba II lines. We adopted the HFS provided by McWilliam (1998). The Eu line list is the same as in Mucciarelli et al. (2008), who adopted the values from Lawler et al. (2001c). We also checked the difference between the HFS provided by Kurucz (1993) and Lawler et al. (2001c). The derived abundances agreed well. The $g f$ values of the La lines were taken from Lawler et al. (2001a), and the HFS provided by Ivans et al. (2006) were also considered. According to McWilliam et al. (1995), HFS is important for any La lines with equivalent widths (EW) greater than $\log _{10}(E W / \lambda)>-5.6$. Hence many of the lines used are probably affected by HFS. The atomic data for the other lines comes from the Vienna Atomic Line Database (VALD). The HFS of Pr and $\mathrm{Yb}$ lines provided by Ivarsson et al. (2001), Sneden et al. (2009), Yb, Biémont et al. (1998), and Sneden et al. (2009), respectively were also adopted. The Yb II abundances are based on the 3694.192 A line. We did not use the Yb II 3289.367 $\AA$ line, because it shows blending from other atomic lines (e.g. V II and $\mathrm{Fe}$ II). We derived the $\mathrm{Ce}, \mathrm{Nd}$, and $\mathrm{Y}$ abundances from weak narrow lines. They probably have no significantly resolved structure because of HFS at the observed spectral resolution.

The selected lines are listed in Tables 5 and 6, along with the transition information and references to the adopted $g f$-values.

\section{Abundance results}

We derived abundances for 39 elements. When elemental abundances for a species were derived from multiple lines, we adopted the the error of the mean (i.e., $\sigma(\log (\epsilon)) / \sqrt{(N)})$ as the uncertainty of the abundance measurement of the species. For the spectrum synthesis measurement, we estimated the uncertainty based on the Cayrel formula (Cayrel 1988), yielding $0.02-0.12$ dex at the $S / N$ of the red and blue spectrum, respectively, and 0.2 dex for lines detected only in the snapshot spectrum. The total errors in $[\mathrm{X} / \mathrm{Fe}]$ for each element are about $0.02-0.30 \mathrm{dex}$, taking into account uncertainties of $150 \mathrm{~K}$ in $T_{\mathrm{eff}}$, and $0.5 \mathrm{dex}$ in $\log g$, which were estimated using a weighted mean of the various estimates listed in Table 3 . In Table 4 we list the mean abundances $(\log \varepsilon)$, the mean errors $\left(\sigma_{\log \varepsilon}\right)$, the number of lines used to determine the mean abundances, and the abundances relative to iron $([\mathrm{X} / \mathrm{Fe}])$. We adopted the solar abundance of Grevesse \& Sauval (1998).

\section{Abundance pattern of HE 1405-0822}

\section{1. n-capture elements}

HE 1405-0822 is a carbon-enhanced metal-poor (CEMP, for the definition see Beers \& Christlieb 2005) r+s star, whose n-capture elements exhibit a high overabundance relative to $\mathrm{Fe}$ and the abundance ratios in the Sun. The only exception is $\mathrm{Sr}$, which is underabundant $([\mathrm{Sr} / \mathrm{Fe}]=-0.18)$. For this star, the ratio of

2 http://kurucz.harvard.edu/linelists/linesmol/ 
Table 4. Summary of the abundances of HE 1405-0822.

\begin{tabular}{|c|c|c|c|c|c|c|}
\hline$Z$ & Species & $N_{\text {lines }}$ & $\log \epsilon$ & $\sigma_{\log \varepsilon}$ & {$[\mathrm{X} / \mathrm{Fe}]$} & Notes \\
\hline 3 & Li I & 2 & 0.90 & 0.20 & & Synth \\
\hline 4 & Be II & 2 & $<-2.80$ & 1.00 & -1.72 & Synth \\
\hline 6 & $\mathrm{CH}$ & 1 & 7.96 & 0.10 & 1.97 & Synth \\
\hline 6 & $\mathrm{C}_{2}$ & 1 & 7.96 & 0.12 & 1.97 & Synth \\
\hline 7 & $\mathrm{NH}$ & 1 & 6.80 & 0.15 & 1.34 & Synth \\
\hline 7 & $\mathrm{CN}$ & 1 & 6.80 & 0.15 & 1.34 & Synth \\
\hline 8 & $\mathrm{OH}$ & 2 & 7.60 & 0.20 & 1.27 & Synth \\
\hline 11 & $\mathrm{Na} \mathrm{I}$ & 2 & 4.62 & 0.15 & 0.73 & Synth \\
\hline 12 & Mg I & 10 & 5.55 & 0.12 & 0.41 & EW\&synth \\
\hline 13 & $\mathrm{Al} \mathrm{I}$ & 2 & 3.07 & 0.10 & -0.99 & Synth \\
\hline 14 & Si I & 3 & 4.55 & 0.14 & -0.58 & EW\& synth \\
\hline 20 & $\mathrm{Ca} \mathrm{I}$ & 11 & 4.26 & 0.10 & 0.35 & EW \\
\hline 21 & Sc II & 3 & 1.15 & 0.10 & 0.48 & EW \\
\hline 22 & Ti I & 9 & 2.85 & 0.10 & 0.32 & EW \\
\hline 22 & Ti II & 9 & 2.99 & 0.10 & 0.32 & EW \\
\hline 23 & V II & 3 & 1.56 & 0.24 & -0.03 & Synth \\
\hline 24 & $\mathrm{Cr} \mathrm{I}$ & 18 & 3.16 & 0.10 & -0.09 & EW\&synth \\
\hline 25 & Mn I & 3 & 2.61 & 0.25 & -0.49 & Synth \\
\hline 25 & Mn II & 8 & 2.61 & 0.12 & -0.49 & Synth \\
\hline 26 & Fe I & 74 & 5.13 & 0.10 & & EW \\
\hline 26 & Fe II & 6 & 5.13 & 0.10 & & EW \\
\hline 27 & Co I & 2 & 2.36 & 0.17 & -0.14 & Synth \\
\hline 28 & Ni I & 5 & 4.00 & 0.12 & 0.18 & EW\&synth \\
\hline 29 & $\mathrm{Cu} \mathrm{I}$ & 2 & 0.70 & 0.11 & -1.17 & Synth \\
\hline 30 & $\mathrm{Zn} \mathrm{I}$ & 2 & 2.50 & 0.02 & 0.25 & EW \\
\hline 38 & Sr II & 2 & 0.32 & 0.04 & -0.18 & Synth \\
\hline 39 & Y II & 13 & 0.10 & 0.10 & 0.30 & Synth \\
\hline 40 & $\mathrm{Zr} \mathrm{II}$ & 16 & 0.97 & 0.11 & 0.80 & Synth \\
\hline 41 & $\mathrm{Nb}$ II & 2 & 0 & 0.3 & 0.98 & Synth \\
\hline 56 & Ba II & 7 & 1.73 & 0.17 & 1.95 & Synth \\
\hline 57 & La II & 13 & 0.26 & 0.16 & 1.47 & Synth \\
\hline 58 & Ce II & 15 & 0.15 & 0.12 & 0.95 & Synth \\
\hline 59 & Pr II & 8 & -0.19 & 0.13 & 1.44 & Synth \\
\hline 60 & Nd II & 17 & 0.70 & 0.12 & 1.63 & Synth \\
\hline 62 & Sm II & 5 & -0.31 & 0.20 & 1.13 & Synth \\
\hline 63 & Eu II & 2 & -0.33 & 0.20 & 1.54 & Synth \\
\hline 64 & Gd II & 5 & -0.19 & 0.20 & 1.12 & Synth \\
\hline 65 & Tb II & 1 & -0.98 & 0.20 & 1.08 & Synth \\
\hline 66 & Dy II & 7 & -0.19 & 0.20 & 1.07 & Synth \\
\hline 68 & Er II & 1 & -0.23 & 0.25 & 1.22 & Synth \\
\hline 70 & Yb II & 2 & 0.40 & 0.20 & 1.86 & Synth \\
\hline 71 & Lu II & 3 & -1.07 & 0.20 & 1.22 & Synth \\
\hline 72 & Hf II & 12 & 0.09 & 0.11 & 1.76 & Synth \\
\hline 82 & $\mathrm{~Pb} \mathrm{I}$ & 2 & 1.96 & 0.20 & 2.30 & Synth \\
\hline
\end{tabular}

$[\mathrm{La} / \mathrm{Eu}]$, which is a good indicator of the s- and r-process contribution in stars, is -0.07 . Indeed, $75 \%$ of the solar La is synthesized by the s-process, while about $97 \%$ of the solar Eu originates from the r-process (Burris et al. 2000). Judging from its Eu and $\mathrm{La}$ abundance ratios, $[\mathrm{Eu} / \mathrm{Fe}]=1.54$ and $[\mathrm{La} / \mathrm{Eu}]=-0.07$, HE 1405-0822 has experienced a major r-process-enrichment event.

Figure 1 shows neither the scaled solar r-pattern nor the scaled solar s-pattern agrees with the neutron-capture element abundance pattern of HE 1405-0822. The two ratios [hs/ls] ${ }^{3}$ and $[\mathrm{Pb} / \mathrm{hs}]$ are good indicators of the ${ }^{13} \mathrm{C}$-pocket efficiency in AGB stars, which are independent of the efficiency of the third dredge-up (TDU) event as well as of the dilutions of the s-process synthetic material both in the AGB envelope and in the secondary of the binary system. The abundance ratios [hs/ls]

\footnotetext{
3 Here we adopted the average of $\mathrm{Ba}$ and $\mathrm{La}$ as "hs", representing the second s-process peak, and the average of $\mathrm{Y}$ and $\mathrm{Zr}$ as "ls", representing the first s-process peak.
}

and $[\mathrm{Pb} / \mathrm{hs}]$ of $\mathrm{HE} 1405-0822$ are 1.16 and 0.59 . The ${ }^{13} \mathrm{C}$-pocket in the AGB star is clearly highly efficient. This is responsible for the significant enhancement of heavy s-process elements such as $\mathrm{Ba}, \mathrm{La}$, and $\mathrm{Pb}$. But, this star does not belong to the so-called lead stars ([Pb/hs] $\geq 1.0$, see Gallino et al. 1998; Goriely \& Mowlavi 2000; Goriely \& Siess 2001) such as HD 187861, HD 224959, or HD 196944 (Van Eck et al. 2001). This means that the efficiency of the ${ }^{13} \mathrm{C}$-pocket is not high enough to provide sufficient neutrons for a large number of $\mathrm{Pb}$ nuclei, which are close to the termination point of the s-process path. In addition, because the ${ }^{22} \mathrm{Ne}$ neutron source mainly contributes to the first s-process peak, the negative [ $\mathrm{Sr} / \mathrm{Fe}]$ ratio in $\mathrm{HE} 1405-0822$ indicates that its former AGB companion probably had a relatively low mass of $M<3 M_{\odot}$ (Bisterzo et al. 2010) or $M<4 M_{\odot}$ (Karakas \& Lattanzio 2007), where the ${ }^{22} \mathrm{Ne}$ neutron source works only marginally for the s-process during the thermal pulses during the AGB phase.

\subsection{Possible formation mechanism}

We compared the observed abundance distribution of HE 1405-0822 with the results of theoretical r- and s-process nucleosynthesis calculations. We used the parametric model for metal-poor stars presented by Zhang et al. (2006) and developed by Cui et al. (2007) and Cui et al. (2010). In the model, we calculated the envelope abundance $N_{i}$ of the ith element as follows:

$N_{i}(Z)=C_{\mathrm{s}} N_{i, \mathrm{~s}}+C_{\mathrm{r}} N_{i, \mathrm{r}} 10^{[\mathrm{Fe} / \mathrm{H}]}$,

where $Z$ is the metallicity of the star, $N_{i, \mathrm{~s}}$ and $N_{i, \mathrm{r}}$ are the abundance of the $i$ th element produced by the s- and r-process (per $\mathrm{Si}=10^{6}$ at $Z=Z_{\odot}$ ) and $C_{\mathrm{s}}$ and $C_{\mathrm{r}}$ are the component coefficients representing the contributions of the s- and the r-process. We assumed that HE 1405-0822 formed from a gas cloud that was enriched by an r-process nucleosynthesis event. Thus, the scaled solar r-element abundance was adopted as the initial abundance $N_{i, \mathrm{r}} . N_{i, \mathrm{~s}}$ was calculated from the parametric model by means of an extensive reaction network described earlier (Liang et al. 2000). Because in s-rich stars the weak r-process only marginally contributes to the production of light n-capture elements such as Sr, Y, and Zr (Liang et al. 2012) compared with the s-process, we ignored the weak r-process contribution in this work.

In Fig. $2 \mathrm{ST}$ refers to the standard case of the ${ }^{13} \mathrm{C}$-pocket including about $3.0 \times 10^{-6} M_{\odot}$ of ${ }^{13} \mathrm{C}$ and $9.0 \times 10^{-8} M_{\odot}$ of ${ }^{14} \mathrm{~N}$ adopted by Gallino et al. (1998), which can reproduce the s-process main component of the solar system using an AGB model with $Z_{\odot} / 2$. The parameter $d i l$ is the dilution factor; i.e., the degree of dilution of the AGB material after accretion by the lower-mass companion in a binary system. This low-mass companion is the star that we observe today. $[\mathrm{r} / \mathrm{Fe}]$ is the degree of initial r-process enrichment in the gas cloud from which the binary formed.

From Fig. 2 we can see that most of the 19 observed heavy neutron-capture elements agree with our theoretical predictions within the measurement uncertainties of the abundances. The sprocess ratios observed in $\mathrm{HE} 1405-0822,[\mathrm{~Pb} / \mathrm{hs}]=0.59$ and [hs $/ \mathrm{ls}]=1.16$, also agree with the predictions of the parametric method within the errors; these predictions are $[\mathrm{Pb} / \mathrm{hs}]=$ 0.69 and $[\mathrm{hs} / \mathrm{ls}]=1.21$. This strongly supports the reliability of our obtained nucleosynthesis parameters, i.e., the neutron exposure per thermal pulse $\Delta \tau=0.69 \mathrm{mbarn}^{-1}$, the overlap factor $r=0.49$, the component coefficient of the s-process 
W. Y. Cui et al.: Abundance analysis of HE 1405-0822. VIII.
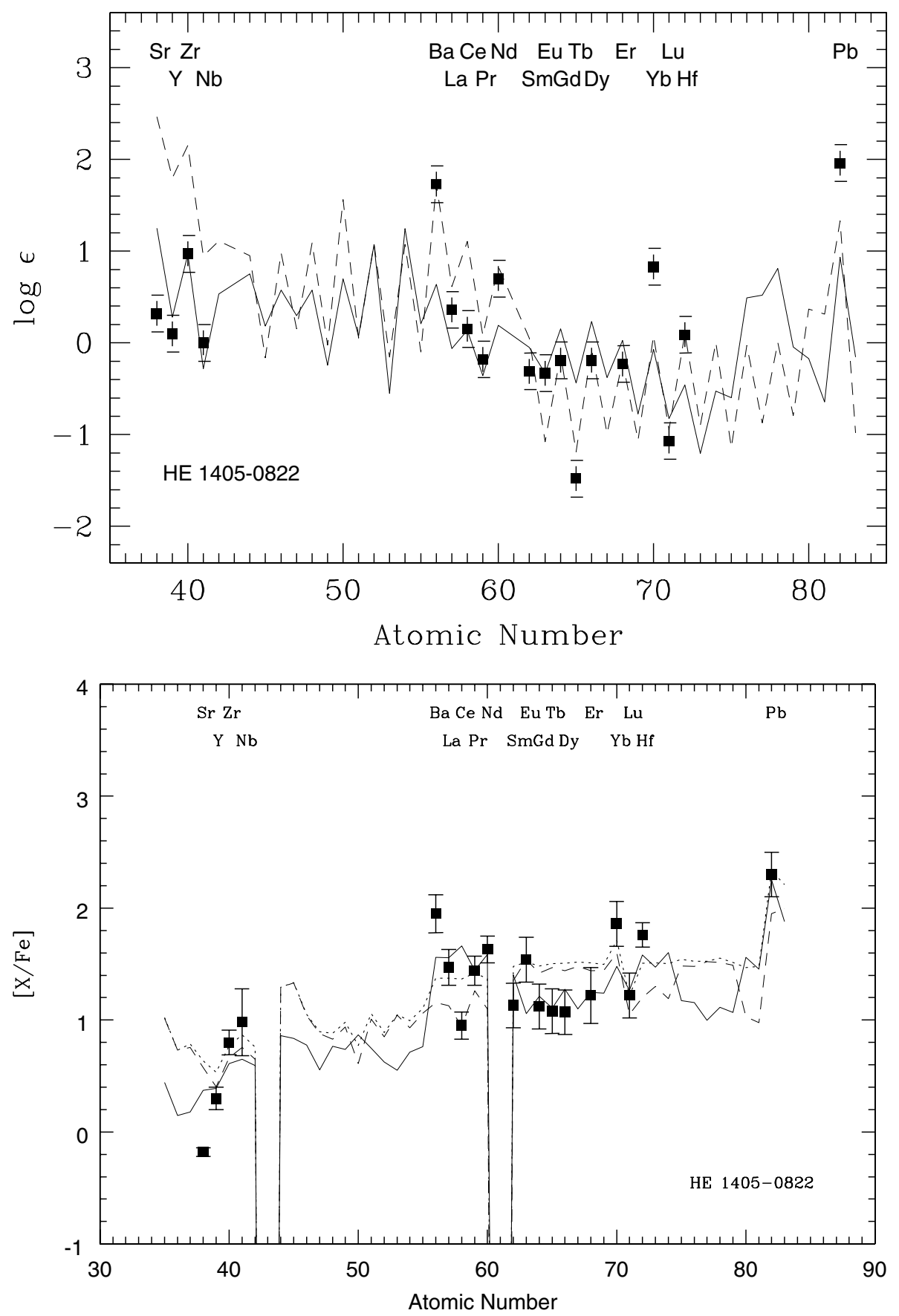

Fig. 1. Observed neutron-capture element abundances of HE 1405-0822 (full squares) compared with the scaled solar s- and r-process abundance patterns (dashed and solid lines, respectively). The solar s-process pattern was normalized to $\mathrm{Ba}$, the solar r-process pattern was normalized to $\mathrm{Eu}$.

Fig. 2. Abundance pattern of HE 1405-0822 (full squares) compared with theoretical predictions. The solid line represents our results based on a parametric method (Zhang et al. 2006; Cui et al. 2010). The other two lines represent the results of two AGB models (Bisterzo et al. 2010) with $[\mathrm{Fe} / \mathrm{H}]=-2.6$, i.e. $2.0 M_{\odot}$, $\mathrm{ST} / 6,[\mathrm{r} / \mathrm{Fe}]=0.9$, dil $=1.4$ (dashed line), and $1.4 M_{\odot}, \mathrm{ST} / 6,[\mathrm{r} / \mathrm{Fe}]=1.0$, dil $=1.1($ dotted line).

$C_{\mathrm{S}}=0.00095$, and the component coefficient of the r-process $C_{\mathrm{r}}=8.7$, where the overlap factor $r$ is the fraction of material in the He intershell of an AGB star that still has to experience subsequent neutron exposures. $C_{\mathrm{r}}$ and $C_{\mathrm{s}}$ are the component coefficients that correspond to the s- and r-process contributions.

The mean neutron exposure for HE $1405-0822$ is $\tau_{0}=$ $1.81\left(T_{9} / 0.348\right)^{1 / 2}$, where $T_{9}=0.1$ (in units of $10^{9} \mathrm{~K}$ ). Käppeler et al. (1989) found $\tau_{0}=0.30\left(T_{9} / 0.348\right)^{1 / 2}$ when they fit the solar main component. Based on the primary nature of the ${ }^{13} \mathrm{C}$ source, Gallino et al. (1998) found a maximum neutron exposure of 0.40-0.45 mbarn $^{-1}$ with their standard AGB model, which can reproduce the solar s-process distribution well. Furthermore, they also pointed out that the average s-process efficiency will indeed increase toward lower metallicity, which is mainly due to the decreasing iron abundance, and therefore higher neutronto-seed ratio. The values of $\Delta \tau$ and $\tau_{0}$ for HE 1405-0822 are significantly higher than those for the solar system. This can naturally explain why the enrichment of the s-process material in HE 1405-0822 is significantly stronger than in the solar system.

The overlap factor for HE 1405-0822, $r=0.49$, lies in the range of $r \sim 0.4-0.7$ found by Gallino et al. (1998), using their standard low-mass AGB model at solar metallicity. Using an s-process parametric model without adopting any specific stellar model, Aoki et al. (2001) reported a neutron exposure per pulse of about $0.7-0.8 \mathrm{mbarn}^{-1}$, and a small overlap factor of $\sim 0.1$ for two carbon-rich metal-poor $\mathrm{r}+\mathrm{s}$ stars, LP 625-44 and LP 706-7, with $[\mathrm{Fe} / \mathrm{H}]=-2.7$. That is, these two stars have similar values of the neutron exposure per pulse as HE 1405-0822, but significantly lower values of the overlap factor than HE 1405-0822. Aoki et al. (2001) proposed a new mechanism for the s-process, a single neutron-exposure event. They found that during the first neutron exposure almost all elements except $\mathrm{Pb}$ can be produced in their parametric model. Even the $\mathrm{Pb}$ abundance can be reproduced after about three recurring neutron exposures, 
which corresponds to a small overlap factor of $r \lesssim 0.2$. In conclusion, a single neutron-exposure event of the s-process for HE 1405-0822 can be excluded.

Because HE 1405-0822 is in its red giant evolution phase, the s-elements cannot be synthesized by themselves. Instead, the s-elements were probably synthesized by its former AGB companion in a binary system and were then transferred to its surface by a stellar wind. The component coefficient of the s-process, $C_{\mathrm{s}}=0.00095$, is very small. Therefore, the binary system probably had a long orbital period, which results in a small amount of material that is accreted.

Regarding the origin of the r-process component of the abundance pattern of HE 1405-0822, the accretion-induced collapse mechanism (Qian \& Wasserburg 2003; Cohen et al. 2003) can be excluded for a long orbital period, because it makes the opposite mass accretion difficult to imagine, i.e. the white dwarfs (the remnant of its AGB companion) accreting material from the secondary star observed now. Interestingly, our parametric calculation fitted almost all r-process element abundances such as $\mathrm{Gd}$ (r-process fraction in the solar system $82 \%$; Burris et al. 2000), Tb (94\%), Dy (88\%), Er (84\%), and Lu (79\%), but underestimated the Eu (97\%) and $\mathrm{Yb}(68 \%)$ abundance. The predictions of low-mass AGB model (Bisterzo et al. 2010) with two different initial masses (see dashed and dotted lines) where the r-process pre-enrichment scenario (formed from a cloud which have been polluted by $\mathrm{SNe}$ of type II) were adopted are also plotted in Fig. 2 for comparison. For the low-mass AGB model calculation, the r-element pre-enriched mechanism was adopted, that is, we adopted solar r-element abundances, which scaled to $\mathrm{Eu}$ of HE 1405-0822 as the initial model values. From Fig. 2 we can see that most r-process elements of HE 1405-0822 are overestimated except for Eu and $\mathrm{Yb}$.

We adopted the solar r-process pattern to calculate the main r-process contribution in all model calculations discussed above. However, the abundance pattern of the r-process contribution in HE $1405-0822$ is inconsistent with the scaled solar pattern, therefore it is also inconsistent with the universal pattern observed in r-II stars (Sneden et al. 2008, and references therein). This is in stark contrast to what was found for instance in the r+s star HE 0338-3945 (Cui et al. 2010). Compared with the solar r-process pattern, the incongruously high Eu abundance of HE 1405-0822 relative to other second-r-process-peak elements such as Gd and Tb may be caused by observational uncertainties. If this is not the case, a more complex origin for the r-process is implied, especially for the $\mathrm{r}+\mathrm{s}$ stars.

Lugaro et al. (2012) studied many CEMP-s and CEMP-r+s stars with their detailed AGB evolution models. They found that the r-process pre-enrichment scenario mainly have three problems for explaining the formation of CEMP-r+s stars. (1) They were unable to reproduce the linear correlation observed between $\mathrm{Ba}$ and $\mathrm{Eu}$ enrichments in the currently known sample of CEMP-r+s stars, because the initial $[\mathrm{r} / \mathrm{Fe}]$ value does not affect the final $[\mathrm{Ba} / \mathrm{Fe}]$ value in an AGB model. In other words, in the pre-enrichment scenario the two independent nucleosynthesis processes (i.e., $r$ - and s-process) who do not affect each other cannot reproduce the Ba-Eu-enrichments correlation in CEMP$\mathrm{r}+\mathrm{s}$ stars. (2) It is difficult to explain the smaller number of r-II stars (about 10) compared with CEMP-r+s stars (about 30), because in this scenario CEMP-r+s stars should be formed from r-II stars. (3) Because of the similar r-elements origin in this scenario for $\mathrm{r}-\mathrm{II}$ and CEMP-r+s stars, the different metallicity distribution of $r-I I$ stars at $[\mathrm{Fe} / \mathrm{H}] \simeq-2.8$ and CEMP-r+s stars at $[\mathrm{Fe} / \mathrm{H}] \simeq-2.5$ is difficult to explain. Thus, they argued that the $\mathrm{r}$-process seen in $\mathrm{r}+\mathrm{s}$ stars is different from that observed in r-II stars, and that even the results of s-process nucleosynthesis seen in $\mathrm{r}+\mathrm{s}$ stars is different from that seen in CEMP-s stars because of their typically higher Ba abundances. Because the pre-enrichment scenario seems difficult to determine the origin of the r-elements in $\mathrm{r}+\mathrm{s}$ stars, some other forms of nucleosynthesis must be responsible. To explain this, Lugaro et al. (2012) assumed an "s/r" neutron-capture process, which they described as a single process with features that are similar to or an addition of the s- and r-process. If this is true, it should produce the positive correlations between $\mathrm{Ba}$ and $\mathrm{Eu}$ abundances in $\mathrm{r}+\mathrm{s}$ stars and possibly r-process patterns different from that of the Sun. However, this hypothesis still needs theoretical confirmation. These authors also considered a model involving a stable triple stellar system (for details see Jonsell et al. 2006), despite the unstability problem of the dynamics and the low occurrence likelihood. In the triple system, the primary exploded as an SNe of type II (hereafter SN II) and produced r-elements, and the other companion polluted the observed star during its AGB phase with s-rich material. Such scenarios may offer a solution for the r-process origin of HE 1405-0822. We also cannot exclude the scenario in which the binary system formed from a gas cloud that was enriched with r-process material. But this would imply that the enrichment event would have resulted in an abundance pattern that at least in some cases is different from the r-process pattern seen in the Sun and r-II stars.

We did not include NLTE corrections for any of the neutroncapture elements, but many lines used in the analysis may need NLTE corrections. For Sr II line $4077 \AA$, based on Bergemann et al. (2012) and Belyakova \& Mashonkina (1997), we found that the NLTE corrections for HE $1405-0822$ is about -0.01 dex. However, Mashonkina \& Gehren (2000) reported positive NLTE corrections for the Eu II resonance line at $4129 \AA$ and the subordinate line at $6645 \AA$, which was confirmed by Asplund (2005). Based on Mashonkina et al. (2012), the NLTE corrections for the Eu II lines 4129 and $4205 \AA$ of HE $1405-0822$ are probably about 0.1 dex. The NLTE corrections for $\mathrm{PbI}$ is very high, 0.5-0.6 dex (Mashonkina et al. 2012).

\subsection{Elements up to the iron peak}

Like many other $\mathrm{r}+\mathrm{s}$ stars, HE 1405-0822 also exhibits strong enhancements of carbon, nitrogen, and oxygen. In this star, sodium and magnesium are also enhanced. Because we cannot calculate the abundances of the elements up to the iron peak with our parametric method for the s-process, we only compared the observed abundances with the AGB model yields of Bisterzo et al. (2010) and the observed abundances of CS 22892-052 (Sneden et al. 2003) which normalized to the iron abundance of HE $1405-0822$.

CS $22892-052$ is an r-II star. Its heavy neutron-captureelement $(Z \geq 56)$ abundances agree well with a scaled solar r-process abundance pattern. Generally, SN II are thought to be responsible for the production of heavy r-process pattern, because of the low metallicities $([\mathrm{Fe} / \mathrm{H}] \sim 3.0)$ of the observed r-II stars, which indicates that the r-process sites must be shortlived and have evolved rapidly, so that the interstellar medium (ISM) could be enriched in r-elements prior to the formation of the r-II stars. Indeed, the enhanced $\alpha$-elements such as $\mathrm{O}, \mathrm{Mg}$, and $\mathrm{Si}$ in CS 22892-052 are thought to be generated by the pollution by SN II. From Fig. 3 we can see that the observed abundances of the elements from $\mathrm{Ca}$ to $\mathrm{Zn}$ in HE 1405-0822 agree well with the scaled ones of CS 22892-052 and also with the predictions of Bisterzo et al. (2010). This means that the 
W. Y. Cui et al.: Abundance analysis of HE 1405-0822. VIII.

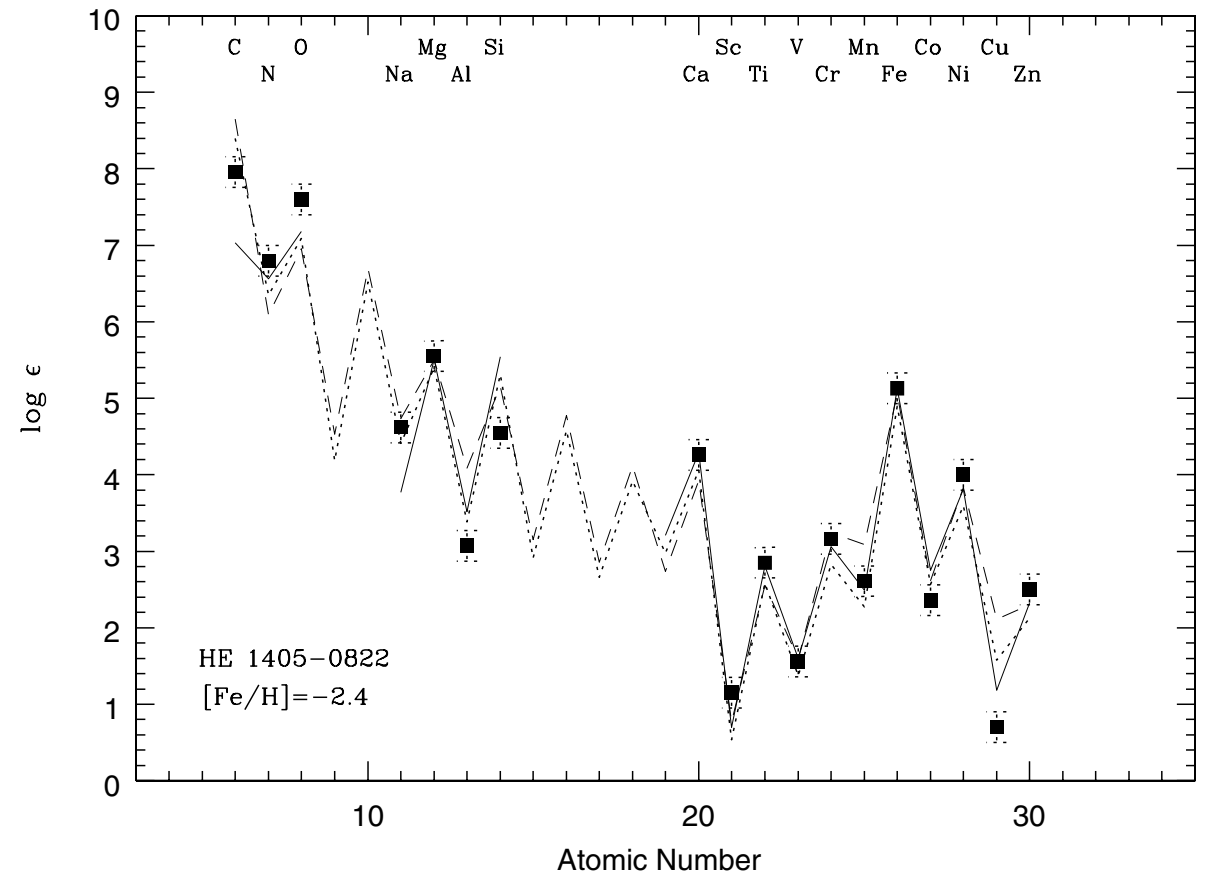

Fig. 3. Abundance pattern of HE 1405-0822 (full squares) compared with theoretical predictions. The solid line represents the observed distribution of CS 22892-052 normalized to iron. The other two lines represent the results of AGB models (Bisterzo et al. 2010) with $[\mathrm{Fe} / \mathrm{H}]=-2.6$, i.e. $2.0 M_{\odot}, \mathrm{ST} / 6,[r / \mathrm{Fe}]=0.9$, dil $=1.4$ (dashed line), and $1.4 M_{\odot}, \mathrm{ST} / 6$, $[r / \mathrm{Fe}]=1.0$, dil $=1.1$ (dotted line) . abundances of these elements did not change during the evolution of the binary system, but remained at the values of ISM at the time when and location where HE 1405-0822 formed.

From Fig. 3 we can see that neither the AGB model predictions nor the scaled abundances of CS 22892-052 fit the carbon, nitrogen, and oxygen abundances of HE 1405-0822 well. For the $\mathrm{C}$ and $\mathrm{N}$ abundances of CS 22892-052 and HE 1405-0822, both $\mathrm{CH}$ and $\mathrm{CN}$ features were used. The AGB models of Bisterzo et al. (2010) often overestimate the carbon and oxygen abundance and underestimate the nitrogen abundance compared with stars that enriched in neutron-capture elements (Bisterzo et al. 2011). This may be due to the model itself, for instance for the incorrect yields of $\mathrm{C}, \mathrm{N}$, and $\mathrm{O}$, or the uncertainty of the observed abundances. Due to the strong temperature sensitivity of $\mathrm{CH}$ and $\mathrm{CN}$ molecular lines, the uncertainty of the derived molecular-based $\mathrm{C}$ and $\mathrm{N}$ abundances is large at low metallicity. 3D corrections for $\mathrm{C}$ and $\mathrm{N}$ can reach about -0.5 to -0.3 dex at low metallicity (Asplund \& García Pérez 2001; Asplund 2004). However, since the magnitudes of the 3D corrections of these two elements are roughly the same, the $\mathrm{C} / \mathrm{N}$ ratio is probably not strongly affected. We recall that the $3 \mathrm{D}$ corrections are themselves highly model-dependent and highly uncertain at present. In HE 1405-0822, this ratio is $\mathrm{C} / \mathrm{N}=14$, which strongly suggests that hot-bottom burning (HBB) did not occur in the former AGB companion, because if HBB had occured, the observed $\mathrm{C} / \mathrm{N}$ ratio would be a constant $1 / 15$ (McSaveney et al. 2007) or 1/10 (Herwig 2004). Using detailed evolution models of AGB stars, Karakas \& Lattanzio (2007) showed that the lowest mass limit is between 2.5 and $3 M_{\odot}$, where HBB could set in around $[\mathrm{Fe} / \mathrm{H}]=-2.3$. This is consistent with the low-mass estimate for the former AGB companion of HE 1405-0822 presented in Sects. 5.1 and 5.2.

Non-LTE effects of UV OH molecular line formation may be strong, but NLTE corrections are not available. 3D effects and missing line opacities are thought be an important uncertainty in deriving an abundance from UV-OH lines. García Pérez et al. (2006), however, found a good agreement between the oxygen abundance derived from [O I] and UV-OH lines. According to Herwig (2004) and Sivarani et al. (2006), low-mass AGB also produce some oxygen, which does not change the initial oxygen abundance significantly at higher metallicities. Furthermore, it could increase the oxygen abundances for low metallicity stars. But we can from Fig. 3 see that the high oxygen abundance of HE 1405-0822 can be reached neither by CS 22892-052 nor by AGB predictions (Bisterzo et al. 2011). OH lines were used here for the oxygen abundances of HE 1405-0822, while Sneden et al. (2003) used the [O I] 6300 feature for CS 22892-052. High oxygen abundance is also seen in many $r+s$ stars (Masseron et al. 2010). For the dilution effect, Cui et al. (2010) pointed out that the oxygen abundance contributed by the low-mass AGB stars could only reach $[\mathrm{O} / \mathrm{Fe}] \sim 0.8$, for $[\mathrm{C} / \mathrm{Fe}] \sim 2.0$ based on the AGB model of Karakas \& Lattanzio (2007). The possible origin for most of the oxygen in HE 1405-0822 is probably an SN II, a similar origin as for CS 22892-052.

We also found sodium and magnesium to be enhanced in HE $1405-0822$, i.e. $[\mathrm{Na} / \mathrm{Fe}]=0.73$ and $[\mathrm{Mg} / \mathrm{Fe}]=0.41$. For $\mathrm{Na}$ and $\mathrm{Mg}$ the observations and AGB model predictions in Fig. 3 match well. The scaled Mg abundance of CS 22892-052 agrees well with the Mg abundance of HE 1405-0822, but this is not the case for Na. Again, we need to consider NLTE corrections before we can draw any conclusions. The Na D lines ( $\lambda 5889$ and $\lambda 5895)$ are significantly affected by NLTE, which are used in the present work as well for CS 22898-052. For $\mathrm{Mg}$ abundances, there are at least three common $\mathrm{Mg}$ I features used for both HE 1405-0822 and CS 22892-052. In metal-poor stars, typical corrections for $\mathrm{Na}$ are about -0.3 dex (Andrievsky et al. 2007), and for $\mathrm{Mg}$ about +0.2 dex (Aoki et al. 2007; Andrievsky et al. 2010). Since the stellar parameters are similar for CS 22892-052 and HE 1405-0822, the NLTE corrections of $\mathrm{Na}$ and $\mathrm{Mg}$ are probably also similar for these two stars.

Even taking into account typical NLTE corrections, the enhancement of $\mathrm{Na}$ and $\mathrm{Mg}$ in HE 1405-0822 could still be explained by mass transfer from a lower-mass AGB companion, where the primary ${ }^{22} \mathrm{Ne}$ mainly operated as a neutron poison in the ${ }^{13} \mathrm{C}$-pocket. In low-mass AGB stars of low metallicity $([\mathrm{Fe} / \mathrm{H}]<-1.0)$, a primary production of $\mathrm{Na}$ and $\mathrm{Mg}$ can be generated by the reactions ${ }^{22} \mathrm{Ne}(n, \gamma)^{23} \mathrm{Na}$, and then $\left.\left.{ }^{23} \mathrm{Na}(n, \gamma){ }^{24} \mathrm{Mg},{ }^{22} \mathrm{Ne}(\alpha, n)\right)^{25} \mathrm{Mg},{ }^{22} \mathrm{Ne}(\alpha, \gamma)\right)^{26} \mathrm{Mg}$ (Mowlavi 1999; Gallino et al. 2006). The primary production of ${ }^{22} \mathrm{Ne}$ increases with the initial mass of the AGB star at very low 
metallicity (Bisterzo et al. 2006). The light enrichment of $\mathrm{Mg}$ in HE 1405-0822 also supports a low-mass AGB companion, since there is not enough ${ }^{22} \mathrm{Ne}$ to feed a higher $\mathrm{Mg}$ abundance. In addition, because $[\mathrm{Mg} / \mathrm{Fe}]=0.41$ of $\mathrm{HE} 1405-0822$ is very similar to the value seen in other field stars, a common origin of $\mathrm{Mg}$, that is pre-enriched by SN II (Gehren et al. 2006; Andrievsky et al. 2010), should not be excluded.

In HE $1405-0822, \mathrm{Al}$ is strongly underabundant; $[\mathrm{Al} / \mathrm{Fe}]=$ -0.99 . The NLTE correction is expected to be about 0.15 dex (Andrievsky et al. 2008). If applied, the Al abundance would match the theoretical prediction of an AGB model with $M=$ 1.4 $M_{\odot}$ (Bisterzo et al. 2010). The low abundance of Al also supports our assumption, i.e. a low-mass former AGB companion of HE 1405-0822, which contributed little primary Al (Karakas \& Lattanzio 2007). This means that the observed Al probably also comes from the ISM at the time and place where HE 1405-0822 formed.

The differences between the $\mathrm{Si}$ abundances of HE 1405-0822, the scaled abundances of CS 22892-052, and the AGB model predictions (see Fig. 3) cannot be explained by the small negative NLTE correction of about -0.05 dex determined by Shi et al. (2009, 2011). A possible explanation are different masses and yields of the SN II that pre-enriched HE 1405-0822 and CS 22892-052. Preston et al. (2006) discussed the systematic effects in the Si abundances caused by the use of different lines. Si abundances of HE 1405-0822 are derived from Si I 3905.523 and $4102.936 \AA$ Alines. Sneden et al. (2003) also used the Si I 4102.94 A line for CS 22892-052.

\section{Conclusions}

We have analyzed high-quality VLT/UVES spectra of HE 1405-0822 and derived accurate abundances for 39 elements, including 19 neutron-capture elements. HE 1405-0822 shows strong enhancement in both the r-process and sprocess elements (e.g., $[\mathrm{Eu} / \mathrm{Fe}]=1.54,[\mathrm{Ba} / \mathrm{Fe}]=1.95$, and $[\mathrm{Pb} / \mathrm{Fe}]=2.3)$, therefore we confirm that it is an $\mathrm{r}+\mathrm{s}$ star.

We discussed several scenarios for the origin of the abundance pattern of HE 1405-0822, taking into account the possible influence of NLTE and 3D corrections on the interpretation of our results.

Because HE 1405-0822 is in its red giant evolutionary phase, it cannot produced its strong enhancement of $\mathrm{C}, \mathrm{N}$, and s-elements by itself. Instead, it is very likely that these enhancements were produced in a formerly more massive companion during its AGB phase and were transferred to the surface of the star that we observe today. The binarity of HE 1405-0822 is confirmed by the fact that the star shows significant radial velocity variations. Combining the enriched s-process material and significant radial velocity variations of HE 1405-0822, its preAGB companion probably is a white dwarf now. However, excess UV-flux measurements are also needed to confirm this. In addition, we also need long-term radial velocity monitoring to confirm its binary nature, and to determine its orbital period and parameters.

Neither the scaled solar s-process pattern nor the scaled solar r-process pattern match the observed abundance pattern of HE 1405-0822 well. We compared the abundance pattern with predictions of our parametric method (Zhang et al. 2006; Cui et al. 2010) and two AGB model yields of Bisterzo et al. (2010). In both cases, the $\mathrm{Pb}$ to heavy s-process element ratio of this star $([\mathrm{Pb} / \mathrm{hs}]=0.59)$ and the heavy-to-light s-process element ratio ( $[\mathrm{hs} / \mathrm{ls}]=1.16$ ) can be reproduced by the models. This strongly supports the reliability of the s-process calculations considered in this work. The parameter fits of the models yield the result that the AGB companion probably is a star with relatively low initial mass, about $\leq 2 M_{\odot}$. This is supported by the low $\mathrm{Sr} / \mathrm{Fe}$ ratio of $[\mathrm{Sr} / \mathrm{Fe}]=-0.99$, which means that the ${ }^{22} \mathrm{Ne}$ neutron source had only a mild influence on the s-process that occurred in the former AGB companion.

Unlike in HE 0338-3945, we cannot reproduce the r-process pattern of HE 1405-0822 with the universal, main r-process pattern that does not vary from star to star and agrees with the scaled solar r-process pattern to within measurement uncertainties. If observational uncertainties are not the reason, this suggests that the origin of the heavy neutron-capture elements in the $r+s$ star is more complex than previously expected. A possible solution is the $\mathrm{s} / \mathrm{r}$ neutron-capture process suggested by Lugaro et al. (2012), which is assumed to be a single process with features similar to, or an addition of, the s- and the r-process. If this is true, it is expected to produce the positive correlations between $\mathrm{Ba}$ and $\mathrm{Eu}$ abundances in $\mathrm{r}+\mathrm{s}$ stars, and maybe r-process patterns different from that of the Sun. However, this hypothesis still needs theoretical confirmation. In addition, we also cannot exclude the scenario in which the binary system formed from a gas cloud that was enriched with r-process material. However, this would imply that the enrichment event would have resulted in an abundance pattern that at least in some cases is different from the r-process pattern seen in the Sun and r-II stars.

From the $\mathrm{C} / \mathrm{N}$ ratio of 14 observed in HE 1405-0822, it can be excluded with high confidence that HBB occurred in its former AGB companion. According to the evolutionary models of Karakas \& Lattanzio (2007), the former massive companion probably had an initial mass of less than $3 M_{\odot}$.

The enhanced sodium and magnesium abundances of the star can be fitted well by the AGB model of Bisterzo et al. (2010), who highlighted that the primary ${ }^{22} \mathrm{Ne}$ mainly acted as a neutron poison in the ${ }^{13} \mathrm{C}$-pocket of AGB stars with low mass and metallicity, which could directly result in a significant production of $\mathrm{Na}$ and $\mathrm{Mg}$. Because $[\mathrm{Mg} / \mathrm{Fe}]=0.41$ of $\mathrm{HE} 1405-0822$ is very similar to the value seen in other field stars, a common origin of $\mathrm{Mg}$, that is pre-enriched by SN II (Gehren et al. 2006; Andrievsky et al. 2010), cannot be excluded.

The low aluminum abundance also supports the idea of a low-mass AGB companion of HE 1405-0822, which is consistent with the results obtained from the model of Bisterzo et al. (2010). The light elements from calcium to zinc in HE 1405-0822 agree well with the scaled abundance distribution of these elements seen in CS 22892-052. This indicates that these elements originate from an ISM that was already well mixed at the time when these two stars formed.

Acknowledgements. We heartly thank the anonymous referee for positive and constructive comments which helped to improve this paper greatly. W.Y.C. would like to thank C. J. Hansen, J. Ren, E. Caffau, L. Sbordone, H.-G. Ludwig, K. Andreas and G. Zhao for their friendly help. The authors thank Paul Barklem for the preliminary analysis using his code and T.S. is thankful for his hospitality during the visit at Uppsala, when the early analysis was carried out. This work is supported by Deutsche Forschungsgemeinschaft through grant $\mathrm{CH} 214 / 5-1$ and Sonderforschungsbereich SFB881 "The Milky Way System" (subproject A5), as well as by the Global Networks Program of Universität Heidelberg. W. Y. Cui is also supported by the National Natural Science Foundation of China under grant 11003002, U1231119, 11273011 and 11021504, the Science Foundation of Hebei Normal University under grants L2007B07 and L2009Z04, the Natural Science Foundation of Hebei Province under grants A2011205102, and the Program for Excellent Innovative Talents in University of Hebei Province under grant CPRC034. We made use of model atmosphere from the MARCS library, and the NIST and VALD databases. 


\section{References}

Alonso, A., Arribas, S., \& Martinez-Roger, C. 1996, A\&A, 313, 873 Alvarez, R., \& Plez, B. 1998, A\&A, 330, 1109

Andrievsky, S. M., Spite, M., Korotin, S. A., et al. 2007, A\&A, 464, 1081 Andrievsky, S. M., Spite, M., Korotin, S. A., et al. 2008, A\&A, 481, 481 Andrievsky, S. M., Spite, M., Korotin, S. A., et al. 2010, A\&A, 509, A88 Aoki, W., Ryan, S. G., Norris, J. E., et al. 2001, ApJ, 561, 346

Aoki, W., Beers, T. C., Christlieb, N., et al. 2007, ApJ, 655, 492

Arcones, A., \& Montes, F. 2011, ApJ, 731, 5

Asplund, M. 2004, Mem. Soc. Astron. It., 75, 300

Asplund, M. 2005, ARA\&A, 43, 481

Asplund, M., \& García Pérez, A. E. 2001, A\&A, 372, 601

Barbuy, B., Spite, M., Spite, F., et al. 2005, A\&A, 429, 1031

Barklem, P. S., Christlieb, N., Beers, T. C., et al. 2005, A\&A, 439, 129

Beers, T. C., \& Christlieb, N. 2005, ARA\&A, 43, 531

Beers, T. C., Flynn, C., Rossi, S., et al. 2007, ApJS, 168, 128

Belyakova, E. V., \& Mashonkina, L. I. 1997, AZh, 74, 601

Bergemann, M., Hansen, C. J., Bautista, M., \& Ruchti, G. 2012, A\&A, 546, A90

Biémont, E., Dutrieux, J.-F., Martin, I., \& Quinet, P. 1998, J. Phys. B At. Mol. Phys., 31, 3321

Bisterzo, S., Gallino, R., Straniero, O., et al. 2006, Mem. Soc. Astron. It., 77, 985

Bisterzo, S., Gallino, R., Straniero, O., Cristallo, S., \& Käppeler, F. 2010, MNRAS, 404, 1529

Bisterzo, S., Gallino, R., Straniero, O., Cristallo, S., \& Käppeler, F. 2011, MNRAS, 418, 284

Burbidge, E. M., Burbidge, G. R., Fowler, W. A., \& Hoyle, F. 1957, Rev. Mod. Phys., 29, 547

Burris, D. L., Pilachowski, C. A., Armandroff, T. E., et al. 2000, ApJ, 544, 302

Cameron, A. G. W. 2001, ApJ, 562, 456

Cameron, A. G. W. 2003, ApJ, 587, 327

Cayrel, R. 1988, in The Impact of Very High S/N Spectroscopy on Stellar Physics, eds. G. Cayrel de Strobel, \& M. Spite, IAU Symp., 132, 345

Chiappini, C., Frischknecht, U., Meynet, G., et al. 2011, Nature, 472, 454

Christlieb, N., Beers, T. C., Barklem, P. S., et al. 2004, A\&A, 428, 1027

Christlieb, N., Schörck, T., Frebel, A., et al. 2008, A\&A, 484, 721

Cohen, J. G., Christlieb, N., Qian, Y.-Z., \& Wasserburg, G. J. 2003, ApJ, 588, 1082

Cowan, J. J., Sneden, C., Burles, S., et al. 2002, ApJ, 572, 861

Cowan, J. J., Roederer, I. U., Sneden, C., \& Lawler, J. E. 2011, in RR Lyrae

Stars, Metal-Poor Stars, and the Galaxy, ed. A. McWilliam, 223

Cui, W.-Y., Zhang, B., Ma, K., \& Zhang, L. 2007, ApJ, 657, 1037

Cui, W.-Y., Zhang, J., Zhu, Z.-Z., \& Zhang, B. 2010, ApJ, 708, 51

François, P., Depagne, E., Hill, V., et al. 2007, A\&A, 476, 935

Frebel, A., Christlieb, N., Norris, J. E., et al. 2007, ApJ, 660, L117

Freiburghaus, C., Rosswog, S., \& Thielemann, F.-K. 1999, ApJ, 525, L121

Gallino, R., Arlandini, C., Busso, M., et al. 1998, ApJ, 497, 388

Gallino, R., Bisterzo, S., Husti, L., et al. 2006, in Int. Symp. Nucl. Astrophys. Nuclei in the Cosmos

García Pérez, A. E., Asplund, M., Primas, F., Nissen, P. E., \& Gustafsson, B. 2006, A\&A, 451, 621

Gehren, T., Shi, J. R., Zhang, H. W., Zhao, G., \& Korn, A. J. 2006, A\&A, 451, 1065

Goriely, S., \& Mowlavi, N. 2000, A\&A, 362, 599

Goriely, S., \& Siess, L. 2001, A\&A, 378, L25

Grevesse, N., \& Sauval, A. J. 1998, Space Sci. Rev., 85, 161

Gustafsson, B., Edvardsson, B., Eriksson, K., et al. 2003, in Modelling of Stellar Atmospheres, eds. N. Piskunov, W. W. Weiss, \& D. F. Gray, IAU Symp., 210, $4 \mathrm{P}$

Hansen, C. J., \& Primas, F. 2011, A\&A, 525, L5

Hayek, W., Wiesendahl, U., Christlieb, N., et al. 2009, A\&A, 504, 511 Herwig, F. 2004, ApJS, 155, 651

Hill, V., Plez, B., Cayrel, R., et al. 2002, A\&A, 387, 560

Honda, S., Aoki, W., Ishimaru, Y., Wanajo, S., \& Ryan, S. G. 2006, ApJ, 643, 1180

Honda, S., Aoki, W., Ishimaru, Y., \& Wanajo, S. 2007, ApJ, 666, 1189

Iben, Jr., I., \& Renzini, A. 1983, ARA\&A, 21, 271

Ivans, I. I., Simmerer, J., Sneden, C., et al. 2006, ApJ, 645, 613

Ivarsson, S., Litzén, U., \& Wahlgren, G. M. 2001, Phys. Scr., 64, 455

Izutani, N., Umeda, H., \& Tominaga, N. 2009, ApJ, 692, 1517

Jonsell, K., Barklem, P. S., Gustafsson, B., et al. 2006, A\&A, 451, 651

Käppeler, F., Beer, H., \& Wisshak, K. 1989, Rep. Prog. Phys., 52, 945
Karakas, A., \& Lattanzio, J. C. 2007, PASA, 24, 103

Kratz, K.-L., Farouqi, K., Pfeiffer, B., et al. 2007, ApJ, 662, 39

Kupka, F., Piskunov, N., Ryabchikova, T. A., Stempels, H. C., \& Weiss, W. W. 1999, A\&AS, 138, 119

Kurucz, R. L. 1993, SYNTHE spectrum synthesis programs and line data

Lai, D. K., Bolte, M., Johnson, J. A., et al. 2008, ApJ, 681, 1524

Lattimer, J. M., Mackie, F., Ravenhall, D. G., \& Schramm, D. N. 1977, ApJ, 213,225

Lawler, J. E., Bonvallet, G., \& Sneden, C. 2001a, ApJ, 556, 452

Lawler, J. E., Wickliffe, M. E., Cowley, C. R., \& Sneden, C. 2001b, ApJS, 137, 341

Lawler, J. E., Wickliffe, M. E., den Hartog, E. A., \& Sneden, C. 2001c, ApJ, 563, 1075

Lawler, J. E., Wyart, J.-F., \& Blaise, J. 2001d, ApJS, 137, 351

Liang, Y. C., Zhao, G., \& Zhang, B. 2000, A\&A, 363, 555

Liang, S., Li, H., Shen, X., Cui, W., \& Zhang, B. 2012, PASP, 124, 304

Lucatello, S., Beers, T. C., Christlieb, N., et al. 2006, ApJ, 652, L37

Lucatello, S., Masseron, T., \& Johnson, J. A. 2009, PASA, 26, 303

Lugaro, M., Karakas, A. I., Stancliffe, R. J., \& Rijs, C. 2012, ApJ, 747, 2

Mashonkina, L., \& Gehren, T. 2000, A\&A, 364, 249

Mashonkina, L., Christlieb, N., Barklem, P. S., et al. 2010, A\&A, 516, A46

Mashonkina, L., Ryabtsev, A., \& Frebel, A. 2012, A\&A, 540, A98

Masseron, T., Johnson, J. A., Plez, B., et al. 2010, A\&A, 509, A93

McClure, R. D., \& Woodsworth, A. W. 1990, ApJ, 352, 709

McClure, R. D., Fletcher, J. M., \& Nemec, J. M. 1980, ApJ, 238, L35

McSaveney, J. A., Wood, P. R., Scholz, M., Lattanzio, J. C., \& Hinkle, K. H. 2007, MNRAS, 378, 1089

McWilliam, A. 1998, AJ, 115, 1640

McWilliam, A., Preston, G. W., Sneden, C., \& Searle, L. 1995, AJ, 109, 2757

Mowlavi, N. 1999, A\&A, 350, 73

Mucciarelli, A., Caffau, E., Freytag, B., Ludwig, H.-G., \& Bonifacio, P. 2008, A\&A, 484, 841

North, P., Jorissen, A., \& Mayor, M. 2000, in The Carbon Star Phenomenon, ed. R. F. Wing, IAU Symp., 177, 269

Pignatari, M., Herwig, F., Bennet, M. E., et al. 2008, in Nuclei in the Cosmos (NIC X)

Plez, B., Cohen, J. G., \& Meléndez, J. 2005, in From Lithium to Uranium: Elemental Tracers of Early Cosmic Evolution, eds. V. Hill, P. Francois, \& F. Primas, IAU Symp., 228, 267

Preston, G. W., Sneden, C., Thompson, I. B., Shectman, S. A., \& Burley, G. S. 2006, AJ, 132, 85

Qian, Y.-Z., \& Wasserburg, G. J. 2000, Phys. Rep., 333, 77

Qian, Y.-Z., \& Wasserburg, G. J. 2001, ApJ, 559, 925

Qian, Y.-Z., \& Wasserburg, G. J. 2002, ApJ, 567, 515

Qian, Y.-Z., \& Wasserburg, G. J. 2003, ApJ, 588, 1099

Roederer, I. U., Cowan, J. J., Karakas, A. I., et al. 2010a, ApJ, 724, 975

Roederer, I. U., Sneden, C., Lawler, J. E., \& Cowan, J. J. 2010b, ApJ, 714, L123

Rosswog, S., Liebendörfer, M., Thielemann, F.-K., et al. 1999, A\&A, 341, 499

Schlegel, D. J., Finkbeiner, D. P., \& Davis, M. 1998, ApJ, 500, 525

Shi, J. R., Gehren, T., Mashonkina, L., \& Zhao, G. 2009, A\&A, 503, 533

Shi, J. R., Gehren, T., \& Zhao, G. 2011, A\&A, 534, A103

Sivarani, T., Bonifacio, P., Molaro, P., et al. 2004, A\&A, 413, 1073

Sivarani, T., Beers, T. C., Bonifacio, P., et al. 2006, A\&A, 459, 125

Sneden, C., Cowan, J. J., Lawler, J. E., et al. 2003, ApJ, 591, 936

Sneden, C., Cowan, J. J., \& Gallino, R. 2008, ARA\&A, 46, 241

Sneden, C., Lawler, J. E., Cowan, J. J., Ivans, I. I., \& Den Hartog, E. A. 2009, ApJS, 182, 80

Truran, J. W., Cowan, J. J., Pilachowski, C. A., \& Sneden, C. 2002, PASP, 114, 1293

Van Eck, S., Goriely, S., Jorissen, A., \& Plez, B. 2001, Nature, 412, 793

Van Eck, S., Goriely, S., Jorissen, A., \& Plez, B. 2003, A\&A, 404, 291

Wanajo, S., \& Ishimaru, Y. 2006, Nucl. Phys. A, 777, 676

Wanajo, S., Kajino, T., Mathews, G. J., \& Otsuki, K. 2001, ApJ, 554, 578

Wanajo, S., Tamamura, M., Itoh, N., et al. 2003, ApJ, 593, 968

Wasserburg, G. J., \& Qian, Y.-Z. 2000, ApJ, 529, L21

Wasserburg, G. J., Busso, M., \& Gallino, R. 1996, ApJ, 466, L109

Westin, J., Sneden, C., Gustafsson, B., \& Cowan, J. J. 2000, ApJ, 530, 783

Wisotzki, L., Christlieb, N., Bade, N., et al. 2000, A\&A, 358, 77

Woosley, S. E., Wilson, J. R., Mathews, G. J., Hoffman, R. D., \& Meyer, B. S. 1994, ApJ, 433, 229

Zhang, B., Ma, K., \& Zhou, G. 2006, ApJ, 642, 1075

Zhang, J., Cui, W., \& Zhang, B. 2010, MNRAS, 409, 1068

Zijlstra, A. A. 2004, MNRAS, 348, L23 
Table 5. Line data, equivalent widths, and abundances from the analysis of HE 1405-0822.

\begin{tabular}{|c|c|c|c|c|c|}
\hline Species & $\begin{array}{c}\lambda \\
(\AA)\end{array}$ & $\begin{array}{c}\chi \\
(\mathrm{eV})\end{array}$ & $\log g f$ & $\begin{array}{c}W_{\lambda} \\
(\mathrm{m} \AA)\end{array}$ & $\log \epsilon$ \\
\hline Mg I & 5172.684 & 2.710 & -0.380 & 169.9 & 5.368 \\
\hline $\mathrm{Mg} \mathrm{I}$ & 5183.604 & 2.720 & -0.158 & 204.6 & 5.540 \\
\hline $\mathrm{Si} \mathrm{I}$ & 5948.541 & 5.082 & -1.230 & 16.3 & 5.980 \\
\hline $\mathrm{Ca}$ I & 5265.556 & 2.520 & -0.260 & 40.5 & 4.584 \\
\hline $\mathrm{Ca} \mathrm{I}$ & 5349.465 & 2.710 & -0.310 & 14.5 & 4.229 \\
\hline $\mathrm{Ca} \mathrm{I}$ & 5581.965 & 2.520 & -0.710 & 16.6 & 4.489 \\
\hline $\mathrm{Ca} \mathrm{I}$ & 5588.749 & 2.520 & 0.210 & 53.0 & 4.318 \\
\hline $\mathrm{Ca} \mathrm{I}$ & 5590.114 & 2.520 & -0.710 & 12.1 & 4.329 \\
\hline $\mathrm{Ca} \mathrm{I}$ & 5601.277 & 2.520 & -0.690 & 26.4 & 4.724 \\
\hline $\mathrm{Ca}$ I & 5857.451 & 2.930 & 0.230 & 28.6 & 4.277 \\
\hline $\mathrm{Ca}$ I & 6102.723 & 1.880 & -0.790 & 28.6 & 4.163 \\
\hline $\mathrm{Ca} \mathrm{I}$ & 6122.217 & 1.890 & -0.320 & 63.4 & 4.309 \\
\hline $\mathrm{Ca} \mathrm{I}$ & 6162.173 & 1.900 & -0.090 & 79.0 & 4.353 \\
\hline $\mathrm{Ca} \mathrm{I}$ & 6439.075 & 2.520 & 0.470 & 68.6 & 4.291 \\
\hline Sc II & 5031.021 & 1.360 & -0.400 & 59.5 & 1.201 \\
\hline Sc II & 5526.790 & 1.770 & 0.030 & 51.3 & 1.055 \\
\hline Sc II & 5657.896 & 1.510 & -0.600 & 35.9 & 1.133 \\
\hline Ti I & 4981.731 & 0.840 & 0.500 & 56.7 & 2.925 \\
\hline Ti I & 4991.065 & 0.840 & 0.380 & 57.2 & 3.053 \\
\hline Ti I & 4999.503 & 0.830 & 0.250 & 64.1 & 3.290 \\
\hline Ti I & 5014.187 & 0.000 & -1.220 & 40.1 & 3.437 \\
\hline Ti I & 5014.276 & 0.810 & 0.110 & 40.1 & 2.998 \\
\hline Ti I & 5014.187 & 0.000 & -1.220 & 34.8 & 3.341 \\
\hline Ti I & 5014.276 & 0.810 & 0.110 & 34.8 & 2.902 \\
\hline Ti I & 5192.969 & 0.020 & -1.010 & 31.4 & 3.075 \\
\hline Ti I & 5210.385 & 0.050 & -0.880 & 25.1 & 2.845 \\
\hline Ti II & 3500.340 & 0.122 & -2.020 & 93.7 & 3.081 \\
\hline Ti II & 3504.896 & 1.892 & 0.180 & 116.8 & 3.407 \\
\hline Ti II & 3510.845 & 1.893 & 0.140 & 92.5 & 2.843 \\
\hline Ti II & 4865.612 & 1.116 & -2.810 & 22.6 & 3.079 \\
\hline Ti II & 5185.913 & 1.893 & -1.370 & 37.4 & 2.772 \\
\hline Ti II & 5188.680 & 1.582 & -1.050 & 82.0 & 2.860 \\
\hline Ti II & 5226.543 & 1.566 & -1.230 & 72.6 & 2.850 \\
\hline Ti II & 5336.771 & 1.582 & -1.630 & 50.1 & 2.890 \\
\hline Ti II & 5381.015 & 1.566 & -1.970 & 32.5 & 2.908 \\
\hline Cr I & 5409.772 & 1.030 & -0.720 & 35.9 & 3.145 \\
\hline $\mathrm{Fe} \mathrm{I}$ & 3445.149 & 2.200 & -0.540 & 96.8 & 5.723 \\
\hline $\mathrm{Fe} \mathrm{I}$ & 3490.574 & 0.050 & -1.110 & 144.7 & 5.219 \\
\hline $\mathrm{Fe} \mathrm{I}$ & 3497.841 & 0.110 & -1.550 & 128.2 & 5.370 \\
\hline Fe I & 3765.539 & 3.240 & 0.480 & 83.0 & 5.049 \\
\hline Fe I & 3767.192 & 1.010 & -0.390 & 140.5 & 4.979 \\
\hline $\mathrm{Fe} I$ & 3787.880 & 1.010 & -0.860 & 114.5 & 4.848 \\
\hline $\mathrm{Fe} I$ & 4871.318 & 2.870 & -0.360 & 77.1 & 5.133 \\
\hline $\mathrm{Fe} I$ & 4872.138 & 2.880 & -0.570 & 71.8 & 5.248 \\
\hline Fe I & 4891.492 & 2.850 & -0.110 & 88.3 & 5.094 \\
\hline Fe I & 4903.310 & 2.880 & -0.930 & 37.3 & 4.982 \\
\hline $\mathrm{Fe} I$ & 4918.994 & 2.870 & -0.340 & 72.8 & 5.022 \\
\hline Fe I & 4939.687 & 0.860 & -3.340 & 51.2 & 5.419 \\
\hline $\mathrm{Fe} I$ & 4994.130 & 0.920 & -3.080 & 47.3 & 5.155 \\
\hline $\mathrm{Fe} I$ & 5001.864 & 3.880 & 0.010 & 35.1 & 5.071 \\
\hline $\mathrm{Fe} I$ & 5006.119 & 2.830 & -0.620 & 60.5 & 5.020 \\
\hline Fe I & 5041.756 & 1.490 & -2.200 & 76.0 & 5.419 \\
\hline $\mathrm{Fe} \mathrm{I}$ & 5049.820 & 2.280 & -1.360 & 84.8 & 5.627 \\
\hline Fe I & 5051.635 & 0.920 & -2.800 & 67.6 & 5.221 \\
\hline Fe I & 5068.766 & 2.940 & -1.040 & 51.5 & 5.398 \\
\hline $\mathrm{Fe} I$ & 5074.748 & 4.220 & -0.200 & 30.8 & 5.553 \\
\hline $\mathrm{Fe} \mathrm{I}$ & 5151.911 & 1.010 & -3.320 & 66.3 & 5.807 \\
\hline $\mathrm{Fe} \mathrm{I}$ & 5166.282 & 0.000 & -4.200 & 33.6 & 4.994 \\
\hline $\mathrm{Fe} \mathrm{I}$ & 5171.596 & 1.490 & -1.790 & 77.1 & 5.017 \\
\hline $\mathrm{Fe} I$ & 5191.455 & 3.040 & -0.550 & 71.6 & 5.370 \\
\hline
\end{tabular}

Table 5. continued.

\begin{tabular}{|c|c|c|c|c|c|}
\hline Species & $\begin{array}{c}\lambda \\
(\AA)\end{array}$ & $\begin{array}{c}\chi \\
(\mathrm{eV})\end{array}$ & $\log g f$ & $\begin{array}{c}W_{\lambda} \\
(\mathrm{m} \AA)\end{array}$ & $\log \epsilon$ \\
\hline $\mathrm{Fe} \mathrm{I}$ & 5192.344 & 3.000 & -0.420 & 70.5 & 5.175 \\
\hline $\mathrm{Fe} I$ & 5194.942 & 1.560 & -2.090 & 58.1 & 5.041 \\
\hline $\mathrm{Fe} \mathrm{I}$ & 5216.274 & 1.610 & -2.150 & 46.4 & 4.955 \\
\hline Fe I & 5225.526 & 0.110 & -4.790 & 12.8 & 5.176 \\
\hline $\mathrm{Fe} \mathrm{I}$ & 5232.940 & 2.940 & -0.060 & 80.2 & 4.935 \\
\hline $\mathrm{Fe} I$ & 5254.955 & 0.110 & -4.760 & 19.3 & 5.352 \\
\hline Fe I & 5266.555 & 3.000 & -0.390 & 61.4 & 4.973 \\
\hline $\mathrm{Fe} I$ & 5269.537 & 0.860 & -1.320 & 125.4 & 4.931 \\
\hline $\mathrm{Fe} I$ & 5281.790 & 3.040 & -0.830 & 33.6 & 4.962 \\
\hline $\mathrm{Fe} I$ & 5283.621 & 3.240 & -0.520 & 44.8 & 5.074 \\
\hline $\mathrm{Fe} \mathrm{I}$ & 5302.302 & 3.280 & -0.880 & 42.2 & 5.430 \\
\hline $\mathrm{Fe} \mathrm{I}$ & 5307.361 & 1.610 & -2.990 & 15.1 & 5.111 \\
\hline $\mathrm{Fe} I$ & 5324.179 & 3.210 & -0.240 & 64.9 & 5.112 \\
\hline $\mathrm{Fe} \mathrm{I}$ & 5328.039 & 0.920 & -1.470 & 126.1 & 5.152 \\
\hline $\mathrm{Fe} I$ & 5328.532 & 1.560 & -1.850 & 81.7 & 5.231 \\
\hline $\mathrm{Fe} \mathrm{I}$ & 5339.929 & 3.270 & -0.720 & 32.7 & 5.080 \\
\hline $\mathrm{Fe} I$ & 5369.962 & 4.370 & 0.540 & 34.9 & 5.043 \\
\hline $\mathrm{Fe} I$ & 5371.490 & 0.960 & -1.650 & 117.5 & 5.163 \\
\hline $\mathrm{Fe} \mathrm{I}$ & 5383.369 & 4.310 & 0.640 & 42.2 & 5.016 \\
\hline $\mathrm{Fe} \mathrm{I}$ & 5389.479 & 4.420 & -0.410 & 6.6 & 5.158 \\
\hline $\mathrm{Fe} I$ & 5393.168 & 3.240 & -0.910 & 42.6 & 5.419 \\
\hline $\mathrm{Fe} \mathrm{I}$ & 5397.128 & 0.920 & -1.990 & 94.5 & 4.918 \\
\hline Fe I & 5405.775 & 0.990 & -1.840 & 96.4 & 4.888 \\
\hline $\mathrm{Fe} I$ & 5424.068 & 4.320 & 0.520 & 43.4 & 5.167 \\
\hline $\mathrm{Fe} I$ & 5429.697 & 0.960 & -1.880 & 101.9 & 5.016 \\
\hline $\mathrm{Fe} \mathrm{I}$ & 5434.524 & 1.010 & -2.120 & 85.5 & 4.951 \\
\hline $\mathrm{Fe} I$ & 5446.917 & 0.990 & -1.910 & 94.8 & 4.917 \\
\hline $\mathrm{Fe} I$ & 5455.609 & 1.010 & -2.090 & 111.2 & 5.494 \\
\hline $\mathrm{Fe} \mathrm{I}$ & 5497.516 & 1.010 & -2.850 & 57.1 & 5.148 \\
\hline $\mathrm{Fe} \mathrm{I}$ & 5501.465 & 0.960 & -3.050 & 55.2 & 5.260 \\
\hline $\mathrm{Fe} \mathrm{I}$ & 5506.779 & 0.990 & -2.800 & 56.2 & 5.060 \\
\hline Fe I & 5569.618 & 3.420 & -0.540 & 47.5 & 5.321 \\
\hline $\mathrm{Fe} \mathrm{I}$ & 5572.842 & 3.400 & -0.310 & 54.5 & 5.191 \\
\hline $\mathrm{Fe} I$ & 5576.089 & 3.430 & -1.000 & 24.0 & 5.334 \\
\hline Fe I & 5586.756 & 3.370 & -0.140 & 54.7 & 4.991 \\
\hline $\mathrm{Fe} I$ & 5615.644 & 3.330 & -0.140 & 61.6 & 5.066 \\
\hline $\mathrm{Fe} \mathrm{I}$ & 6136.615 & 2.450 & -1.400 & 46.8 & 5.079 \\
\hline Fe I & 6137.692 & 2.590 & -1.400 & 37.4 & 5.068 \\
\hline $\mathrm{Fe} I$ & 6191.558 & 2.430 & -1.420 & 32.8 & 4.824 \\
\hline $\mathrm{Fe} \mathrm{I}$ & 6213.430 & 2.220 & -2.480 & 15.5 & 5.232 \\
\hline $\mathrm{Fe} \mathrm{I}$ & 6219.281 & 2.200 & -2.430 & 17.4 & 5.219 \\
\hline Fe I & 6230.723 & 2.560 & -1.280 & 44.1 & 5.029 \\
\hline $\mathrm{Fe} I$ & 6252.555 & 2.400 & -1.690 & 34.3 & 5.086 \\
\hline $\mathrm{Fe} I$ & 6393.601 & 2.430 & -1.580 & 37.7 & 5.065 \\
\hline $\mathrm{Fe} \mathrm{I}$ & 6400.001 & 3.600 & -0.520 & 41.5 & 5.354 \\
\hline $\mathrm{Fe} \mathrm{I}$ & 6421.351 & 2.280 & -2.030 & 25.6 & 5.108 \\
\hline $\mathrm{Fe} \mathrm{I}$ & 6430.846 & 2.180 & -2.010 & 35.5 & 5.177 \\
\hline $\mathrm{Fe} \mathrm{I}$ & 6494.980 & 2.400 & -1.270 & 51.1 & 4.947 \\
\hline $\mathrm{Fe}$ II & 5197.577 & 3.230 & -2.230 & 46.6 & 5.156 \\
\hline $\mathrm{Fe}$ II & 5234.625 & 3.220 & -2.150 & 49.3 & 5.109 \\
\hline $\mathrm{Fe}$ II & 5325.553 & 3.220 & -3.220 & 7.2 & 5.080 \\
\hline $\mathrm{Fe}$ II & 6247.557 & 3.890 & -2.330 & 11.2 & 5.082 \\
\hline Fe II & 6432.680 & 2.890 & -3.710 & 9.1 & 5.274 \\
\hline $\mathrm{Fe}$ II & 6456.383 & 3.900 & -2.080 & 25.4 & 5.268 \\
\hline $\mathrm{Zn} \mathrm{I}$ & 4722.153 & 4.030 & -0.338 & 14.0 & 2.522 \\
\hline $\mathrm{Zn} \mathrm{I}$ & 4810.528 & 4.078 & -0.137 & 25.1 & 2.478 \\
\hline
\end{tabular}

Notes. The most important atomic and molecular data, wavelength $\lambda$, excitation potential $\chi, \log g f$, equivalent width $W_{\lambda}$, and abundances $\log \epsilon$ are listed. 
W. Y. Cui et al.: Abundance analysis of HE 1405-0822. VIII.

Table 6. Line data and abundances from the analysis of HE 1405-0822.

\begin{tabular}{|c|c|c|c|c|c|}
\hline Species & $\begin{array}{c}\lambda \\
(\AA) \\
(\AA)\end{array}$ & $\begin{array}{c}\chi \\
(\mathrm{eV})\end{array}$ & $\log g f$ & $\log \epsilon$ & Ref. \\
\hline $\mathrm{Li} \mathrm{I}$ & 6707.761 & 0.00 & -0.009 & 0.90 & VALD \\
\hline Li I & 6707.912 & 0.00 & -0.309 & 0.90 & VALD \\
\hline Be II & 3130.420 & 0.00 & -0.168 & $<-2.86$ & VALD \\
\hline Be II & 3131.065 & 0.00 & -0.468 & $<-2.92$ & VALD \\
\hline $\mathrm{NaI}$ & 5889.951 & 0.00 & 0.117 & 4.56 & VALD \\
\hline $\mathrm{Na} \mathrm{I}$ & 5895.924 & 0.00 & -0.184 & 4.68 & VALD \\
\hline $\mathrm{Mg} \mathrm{I}$ & 3329.919 & 2.71 & -1.930 & 5.47 & VALD \\
\hline $\mathrm{Mg} \mathrm{I}$ & 3332.146 & 2.71 & -1.450 & 5.58 & VALD \\
\hline $\mathrm{Mg} \mathrm{I}$ & 3336.674 & 2.72 & -1.230 & 5.53 & VALD \\
\hline $\mathrm{Mg} \mathrm{I}$ & 3838.290 & 2.72 & -1.530 & 5.53 & VALD \\
\hline $\mathrm{Mg} \mathrm{I}$ & 3878.306 & 4.35 & -0.457 & 5.51 & VALD \\
\hline $\mathrm{Mg} \mathrm{I}$ & 3903.859 & 4.35 & -0.511 & 5.57 & VALD \\
\hline $\mathrm{Mg} \mathrm{I}$ & 4702.991 & 4.35 & -0.666 & 5.53 & VALD \\
\hline $\mathrm{Mg} \mathrm{I}$ & 5528.405 & 4.35 & -0.620 & 5.50 & VALD \\
\hline $\mathrm{Al} \mathrm{I}$ & 3944.006 & 0.00 & -0.623 & 3.11 & VALD \\
\hline $\mathrm{Al} \mathrm{I}$ & 3961.520 & 0.01 & -0.323 & 3.02 & VALD \\
\hline Si I & 3905.523 & 1.91 & -0.743 & 4.57 & VALD \\
\hline Si I & 4102.936 & 1.91 & -2.827 & 4.52 & VALD \\
\hline V II & 3593.327 & 1.13 & -0.509 & 1.57 & VALD \\
\hline V II & 3727.343 & 1.69 & -0.231 & 1.56 & VALD \\
\hline V II & 3732.750 & 1.57 & -0.354 & 1.54 & VALD \\
\hline $\mathrm{CrI}$ & 3578.686 & 0.00 & 0.409 & 3.14 & VALD \\
\hline $\mathrm{Cr} \mathrm{I}$ & 3593.485 & 0.00 & 0.307 & 3.19 & VALD \\
\hline $\mathrm{Cr} \mathrm{I}$ & 3605.329 & 0.00 & 0.197 & 3.14 & VALD \\
\hline $\mathrm{Cr} \mathrm{I}$ & 4254.336 & 0.00 & -0.114 & 3.13 & VALD \\
\hline Cr I & 4274.797 & 0.00 & -0.231 & 3.16 & VALD \\
\hline Cr I & 4289.717 & 0.00 & -0.361 & 3.13 & VALD \\
\hline $\mathrm{Cr} I$ & 4344.501 & 1.00 & -0.550 & 3.12 & VALD \\
\hline Cr I & 4351.811 & 1.03 & -0.440 & 3.11 & VALD \\
\hline $\mathrm{Cr} I$ & 5204.511 & 0.94 & -0.208 & 3.21 & VALD \\
\hline Cr I & 5206.037 & 0.94 & 0.019 & 3.14 & VALD \\
\hline $\mathrm{Cr} I$ & 5208.425 & 0.94 & 0.158 & 3.12 & VALD \\
\hline Cr I & 5264.153 & 0.97 & -1.290 & 3.19 & VALD \\
\hline $\mathrm{Cr} I$ & 5296.691 & 0.98 & -1.400 & 3.23 & VALD \\
\hline $\mathrm{Cr} \mathrm{I}$ & 5298.272 & 0.98 & -1.150 & 3.20 & VALD \\
\hline $\mathrm{Cr} \mathrm{I}$ & 5345.796 & 1.00 & -0.980 & 3.14 & VALD \\
\hline $\mathrm{Cr} \mathrm{I}$ & 5348.315 & 1.00 & -1.290 & 3.20 & VALD \\
\hline $\mathrm{Cr} \mathrm{I}$ & 5409.784 & 1.03 & -0.720 & 3.15 & VALD \\
\hline Mn I & 4030.753 & 0.00 & -0.470 & 2.59 & VALD \\
\hline Mn I & 4033.062 & 0.00 & -0.618 & 2.67 & VALD \\
\hline Mn I & 4034.483 & 0.00 & -0.811 & 2.56 & VALD \\
\hline Mn II & 3438.971 & 1.17 & -2.100 & 2.64 & VALD \\
\hline Mn II & 3441.985 & 1.78 & -0.360 & 2.60 & VALD \\
\hline Mn II & 3460.315 & 1.81 & -0.640 & 2.47 & VALD \\
\hline Mn II & 3482.904 & 1.83 & -0.840 & 2.62 & VALD \\
\hline Mn II & 3488.675 & 1.85 & -0.950 & 2.66 & VALD \\
\hline Mn II & 3495.833 & 1.86 & -1.300 & 2.68 & VALD \\
\hline Mn II & 3496.807 & 1.83 & -1.790 & 2.63 & VALD \\
\hline Mn II & 3497.525 & 1.85 & -1.430 & 2.58 & VALD \\
\hline Co I & 3518.346 & 1.05 & 0.070 & 2.34 & VALD \\
\hline Co I & 3995.302 & 0.92 & -0.220 & 2.37 & VALD \\
\hline Ni I & 4980.166 & 3.61 & 0.070 & 3.98 & VALD \\
\hline $\mathrm{Ni} I$ & 5035.357 & 3.64 & 0.290 & 4.02 & VALD \\
\hline $\mathrm{Ni} \mathrm{I}$ & 5137.070 & 1.68 & -1.990 & 3.97 & VALD \\
\hline $\mathrm{Ni}$ I & 5476.900 & 1.83 & -0.890 & 4.08 & VALD \\
\hline $\mathrm{Ni} I$ & 5709.539 & 1.68 & -2.170 & 3.99 & VALD \\
\hline
\end{tabular}

Notes. The most important atomic data, wavelength $\lambda$, excitation potential $\chi$, and $\log g f$ are listed. ${ }^{(*)}$ HFS: is included only for one of transitions listed here. BDM98: Biémont et al. (1998); McW95: McWilliam et al. (1995); McW98: McWilliam (1998); Ivar01: Ivarsson et al. (2001); Ivan06: Ivans et al. (2006); Law01a: Lawler et al. (2001a); Law01b: Lawler et al. (2001b); Law01c: Lawler et al. (2001c); Law01d: Lawler et al. (2001d); Sned09: Sneden et al. (2009); VALD: Kupka et al. (1999); Van03: Van Eck et al. (2003).

\begin{tabular}{|c|c|c|c|c|c|}
\hline Species & $\begin{array}{c}\lambda \\
(\AA)\end{array}$ & $\begin{array}{c}\chi \\
(\mathrm{eV})\end{array}$ & $\log g f$ & $\log \epsilon$ & Ref. \\
\hline $\mathrm{Cu} \mathrm{I}$ & 3247.537 & 0.00 & -0.062 & 0.66 & VALD \\
\hline $\mathrm{Cu} \mathrm{I}$ & 3273.954 & 0.00 & -0.359 & 0.73 & VALD \\
\hline Sr II & 4077.709 & 0.00 & 0.167 & 0.37 & VALD \\
\hline Sr II & 4215.519 & 0.00 & -0.145 & 0.36 & VALD \\
\hline Y II & 3601.919 & 0.10 & -0.180 & 0.00 & VALD \\
\hline Y II & 3774.331 & 0.13 & 0.210 & 0.09 & VALD \\
\hline Y II & 3950.352 & 0.10 & -0.490 & 0.16 & VALD \\
\hline Y II & 3982.594 & 0.13 & -0.490 & 0.06 & VALD \\
\hline Y II & 4374.935 & 0.41 & 0.160 & 0.11 & VALD \\
\hline Y II & 4422.591 & 0.10 & -1.270 & 0.12 & VALD \\
\hline Y II & 4854.863 & 0.99 & -0.380 & 0.10 & VALD \\
\hline Y II & 4883.684 & 1.08 & 0.070 & 0.04 & VALD \\
\hline Y II & 4900.120 & 1.03 & -0.090 & 0.13 & VALD \\
\hline Y II & 5087.416 & 1.08 & -0.170 & 0.10 & VALD \\
\hline Y II & 5200.406 & 0.99 & -0.570 & 0.15 & VALD \\
\hline Y II & 5205.724 & 1.03 & -0.340 & 0.10 & VALD \\
\hline Y II & 5662.925 & 1.94 & 0.160 & 0.10 & VALD \\
\hline $\mathrm{Zr}$ II & 3457.548 & 0.56 & -0.530 & 0.95 & VALD \\
\hline Zr II & 3481.137 & 0.80 & 0.165 & 0.90 & VALD \\
\hline Zr II & 3499.560 & 0.41 & -0.810 & 0.90 & VALD \\
\hline $\mathrm{Zr}$ II & 3551.939 & 0.09 & -0.310 & 1.08 & VALD \\
\hline $\mathrm{Zr}$ II & 3611.889 & 1.74 & 0.450 & 1.07 & VALD \\
\hline Zr II & 3614.765 & 0.36 & -0.252 & 0.90 & VALD \\
\hline $\mathrm{Zr}$ II & 3630.004 & 0.36 & -1.110 & 1.09 & VALD \\
\hline $\mathrm{Zr}$ II & 3668.432 & 0.41 & -1.138 & 0.94 & VALD \\
\hline Zr II & 3714.794 & 0.53 & -0.930 & 0.90 & VALD \\
\hline $\mathrm{Zr}$ II & 3751.606 & 0.97 & 0.012 & 1.02 & VALD \\
\hline Zr II & 3766.795 & 0.41 & -0.812 & 1.09 & VALD \\
\hline $\mathrm{Zr}$ II & 3991.152 & 0.76 & -0.252 & 0.91 & VALD \\
\hline Zr II & 3998.954 & 0.56 & -0.387 & 1.00 & VALD \\
\hline Zr II & 4149.217 & 0.80 & -0.030 & 0.94 & VALD \\
\hline $\mathrm{Zr}$ II & 4208.977 & 0.71 & -0.460 & 0.92 & VALD \\
\hline Zr II & 4496.962 & 0.71 & -0.890 & 0.94 & VALD \\
\hline $\mathrm{Nb}$ II & 3130.780 & 0.44 & 0.410 & -0.00 & VALD \\
\hline $\mathrm{Nb}$ II & 3163.398 & 0.38 & 0.260 & 0.40 & VALD \\
\hline Ba II & 3891.776 & 2.51 & 0.280 & 1.63 & VALD \\
\hline Ba II* & 4130.700 & 2.72 & 0.560 & 1.71 & McW98 \\
\hline Ba II & 4524.925 & 2.51 & -0.360 & 1.69 & VALD \\
\hline Ba II* & 4554.000 & 0.00 & 0.170 & 1.79 & McW98 \\
\hline Ba II & 4899.929 & 2.72 & -0.080 & 1.70 & VALD \\
\hline Ba II* & 4934.100 & 0.00 & -0.150 & 1.66 & McW98 \\
\hline Ba II* & 5853.700 & 0.60 & -1.010 & 1.67 & McW98 \\
\hline Ba II* & 6141.695 & 0.70 & -0.070 & 1.68 & McW98 \\
\hline Ba II & 6496.897 & 0.60 & -0.377 & 1.68 & VALD \\
\hline La II* & 4808.996 & 0.23 & -1.400 & 0.26 & Law01a, Ivan06 \\
\hline La II & 4899.915 & 0.00 & -0.921 & 0.06 & Law01a \\
\hline La II & 4920.976 & 0.13 & -0.730 & 0.26 & Law01a \\
\hline La II & 4921.776 & 0.24 & -0.450 & 0.26 & Law01a \\
\hline La II* & 4970.386 & 0.32 & -1.160 & 0.16 & Law01a, Ivan06 \\
\hline La II* & 4986.819 & 0.17 & -1.300 & 0.06 & Law01a, Ivan06 \\
\hline La II* & 4999.461 & 0.40 & -0.770 & 0.32 & Law01a, Ivan06 \\
\hline La II* & 5114.559 & 0.23 & -1.032 & 0.30 & Law01a, Ivan06 \\
\hline La II* & 5122.988 & 0.32 & -0.850 & 0.31 & Law01a, Ivan06 \\
\hline La II & 5259.379 & 0.17 & -1.950 & 0.30 & Law01a \\
\hline La II & 5290.818 & 0.00 & -1.650 & 0.35 & Law01a \\
\hline La II* & 5303.528 & 0.32 & -1.350 & 0.30 & Law01a, Ivan06 \\
\hline La II* & 5482.268 & 0.00 & -2.230 & 0.33 & Law01a, Ivan06 \\
\hline Ce II & 3655.844 & 0.32 & 0.233 & 0.15 & VALD \\
\hline Ce II & 4042.581 & 0.50 & 0.070 & 0.10 & VALD \\
\hline Ce II & 4053.503 & 0.00 & -0.460 & 0.16 & VALD \\
\hline Ce II & 4120.827 & 0.32 & -0.130 & 0.10 & VALD \\
\hline Ce II & 4137.645 & 0.52 & 0.246 & 0.10 & VALD \\
\hline $\mathrm{Ce}$ II & 4186.594 & 0.86 & 0.813 & 0.15 & VALD \\
\hline $\mathrm{Ce}$ II & 4222.597 & 0.12 & -0.301 & 0.18 & VALD \\
\hline Ce II & 4364.653 & 0.50 & 0.070 & 0.15 & VALD \\
\hline
\end{tabular}


Table 6. continued.

\begin{tabular}{|c|c|c|c|c|c|}
\hline Species & $\begin{array}{c}\lambda \\
(\AA)\end{array}$ & $\begin{array}{c}\underset{(\mathrm{eV})}{ } \\
(\mathrm{e})\end{array}$ & $\log g f$ & $\log \epsilon$ & Ref. \\
\hline $\mathrm{Ce}$ II & 4479.361 & 0.56 & -0.480 & 0.20 & VALD \\
\hline $\mathrm{Ce}$ II & 4486.909 & 0.30 & -0.260 & 0.20 & VALD \\
\hline Ce II & 4523.075 & 0.52 & -0.030 & 0.15 & VALD \\
\hline $\mathrm{Ce}$ II & 4562.359 & 0.48 & 0.230 & 0.13 & VALD \\
\hline $\mathrm{Ce}$ II & 4572.278 & 0.68 & 0.290 & 0.22 & VALD \\
\hline $\mathrm{Ce}$ II & 5187.458 & 1.21 & 0.300 & 0.19 & VALD \\
\hline Ce II & 5274.229 & 1.04 & 0.300 & 0.13 & VALD \\
\hline Pr II & 3908.428 & 0.00 & 0.019 & -0.13 & VALD \\
\hline Pr II & 4100.717 & 0.55 & 0.572 & -0.26 & VALD \\
\hline Pr II* & 4143.120 & 0.37 & 0.609 & -0.13 & Ivar01, Sned09 \\
\hline $\operatorname{Pr}$ II* & 4222.950 & 0.05 & 0.271 & -0.18 & Ivar01, Sned09 \\
\hline Pr II* & 5173.910 & 0.97 & 0.384 & -0.19 & Ivar01, Sned09 \\
\hline Pr II* & 5220.108 & 0.80 & 0.298 & -0.18 & Ivar01, Sned09 \\
\hline $\operatorname{Pr}$ II* & 5259.728 & 0.63 & 0.114 & -0.14 & Ivar01, Sned09 \\
\hline Pr II* & 5322.772 & 0.48 & -0.319 & -0.23 & Ivar01, Sned09 \\
\hline Nd II & 5249.576 & 0.98 & 0.094 & 0.63 & VALD \\
\hline Nd II & 5250.812 & 0.75 & -0.618 & 0.72 & VALD \\
\hline Nd II & 5255.506 & 0.20 & -0.697 & 0.72 & VALD \\
\hline $\mathrm{Nd}$ II & 5273.427 & 0.68 & -0.185 & 0.71 & VALD \\
\hline Nd II & 5293.163 & 0.82 & -0.144 & 0.70 & VALD \\
\hline Nd II & 5303.200 & 0.38 & -1.324 & 0.71 & VALD \\
\hline Nd II & 5311.453 & 0.99 & -0.437 & 0.72 & VALD \\
\hline Nd II & 5319.815 & 0.55 & -0.152 & 0.67 & VALD \\
\hline Nd II & 5336.532 & 0.55 & -1.074 & 0.72 & VALD \\
\hline Nd II & 5356.967 & 1.26 & -0.248 & 0.71 & VALD \\
\hline Nd II & 5361.467 & 0.68 & -0.482 & 0.71 & VALD \\
\hline Nd II & 5385.888 & 0.74 & -0.860 & 0.72 & VALD \\
\hline Nd II & 5485.696 & 1.26 & -0.284 & 0.65 & VALD \\
\hline Nd II & 5594.416 & 1.12 & -0.279 & 0.73 & VALD \\
\hline Nd II & 5688.518 & 0.99 & -0.404 & 0.72 & VALD \\
\hline $\mathrm{Nd}$ II & 5702.238 & 0.75 & -0.744 & 0.72 & VALD \\
\hline Nd II & 5708.271 & 0.86 & -0.581 & 0.70 & VALD \\
\hline Sm II & 4318.927 & 0.28 & -0.268 & -0.34 & VALD \\
\hline Sm II & 4420.524 & 0.33 & -0.383 & -0.32 & VALD \\
\hline Sm II & 4537.941 & 0.49 & -0.230 & -0.28 & VALD \\
\hline Sm II & 4543.943 & 0.33 & -0.577 & -0.29 & VALD \\
\hline Sm II & 4815.805 & 0.19 & -0.775 & -0.35 & VALD \\
\hline Eu II* & 4129.628 & 0.00 & 0.210 & -0.29 & Law01c \\
\hline Eu II* & 4204.908 & 0.00 & 0.220 & -0.33 & Law01c \\
\hline Gd II & 3646.196 & 0.24 & 0.328 & -0.10 & VALD \\
\hline Gd II & 4130.366 & 0.73 & -0.090 & -0.20 & VALD \\
\hline Gd II & 4251.731 & 0.38 & -0.365 & -0.20 & VALD \\
\hline Gd II & 4325.557 & 1.37 & 0.772 & -0.23 & VALD \\
\hline Gd II & 4327.151 & 0.35 & -0.37 & -0.22 & VALD \\
\hline Tb II & 3509.144 & 0.00 & 0.700 & -0.98 & law01b, law01d \\
\hline Dy II & 3694.810 & 0.10 & 0.000 & -0.21 & VALD \\
\hline Dy II & 3836.505 & 0.54 & -0.025 & -0.21 & VALD \\
\hline Dy II & 3898.528 & 0.59 & 0.397 & -0.19 & VALD \\
\hline Dy II & 3944.681 & 0.00 & 0.100 & -0.11 & VALD \\
\hline Dy II & 3978.561 & 0.93 & 0.360 & -0.13 & VALD \\
\hline Dy II & 4077.966 & 0.10 & 0.010 & -0.21 & VALD \\
\hline Dy II & 4957.348 & 0.00 & -1.010 & -0.16 & VALD \\
\hline Er II & 3692.649 & 0.05 & 0.138 & -0.23 & VALD \\
\hline $\mathrm{Yb}^{*} \mathrm{II}$ & 3289.367 & 0.00 & -0.052 & 0.39 & BDM98, Sned09 \\
\hline $\mathrm{Yb}^{*} \mathrm{II}$ & 3694.192 & 0.00 & -0.320 & 0.97 & BDM98, Sned09 \\
\hline Lu II & 3507.380 & 0.00 & -1.160 & -0.91 & VALD \\
\hline Lu II & 4994.126 & 1.54 & -1.320 & -1.05 & VALD \\
\hline Lu II & 5476.676 & 1.76 & -0.276 & -1.05 & VALD \\
\hline Hf II & 3478.980 & 2.16 & 0.280 & 0.18 & VALD \\
\hline Hf II & 3479.289 & 0.38 & -1.040 & 0.07 & VALD \\
\hline Hf II & 3505.219 & 1.04 & -0.080 & 0.06 & VALD \\
\hline Hf II & 3535.549 & 0.61 & -0.540 & 0.03 & VALD \\
\hline Hf II & 3569.034 & 0.79 & -0.400 & 0.08 & VALD \\
\hline
\end{tabular}

Table 6. continued.

\begin{tabular}{lccccc}
\hline \hline Species & $\begin{array}{c}\lambda \\
(\AA)\end{array}$ & $\begin{array}{c}\chi \\
(\mathrm{eV})\end{array}$ & $\log g f$ & $\log \epsilon$ & Ref. \\
\hline Hf II & 3597.394 & 1.89 & -0.030 & 0.00 & VALD \\
Hf II & 3644.352 & 0.79 & -0.480 & 0.13 & VALD \\
Hf II & 3661.045 & 1.87 & -0.320 & 0.10 & VALD \\
Hf II & 3699.731 & 1.67 & -0.300 & 0.08 & VALD \\
Hf II & 3701.156 & 2.16 & 0.330 & 0.18 & VALD \\
Hf II & 3719.276 & 0.61 & -0.870 & 0.08 & VALD \\
Hf II & 3737.869 & 2.34 & 0.160 & 0.07 & VALD \\
Pb I $_{\text {Pb I* }}$ & 3683.462 & 0.97 & -0.460 & 1.93 & VALD \\
\hline
\end{tabular}

